\title{
Exponentially accurate solution tracking for nonlinear ODEs, the higher order Stokes phenomenon and double transseries resummation
}

\author{
C.J. Howls ${ }^{1}$ and A. B. Olde Daalhuis ${ }^{2}$ \\ 1 School of Mathematics, University of Southampton, Highfield, Southampton, \\ SO17 1BJ, UK \\ 2 Maxwell Institute and School of Mathematics, The University of Edinburgh, \\ The King's Buildings, Mayfield Road, Edinburgh, EH9 3JZ, UK \\ E-mail: c.j.howls@maths.soton.ac.uk, A.OldeDaalhuis@ed.ac.uk
}

\begin{abstract}
We demonstrate the conjunction of new exponential-asymptotic effects in the context of a second order nonlinear ordinary differential equation with a small parameter. First, we show how to use a hyperasymptotic, beyond-all-orders approach to seed a numerical solver of a nonlinear ordinary differential equation with sufficiently accurate initial data so as to track a specific solution in the presence of an attractor. Second, we demonstrate the necessary role of a higher order Stokes phenomenon in analytically tracking the transition between asymptotic behaviours in a heteroclinic solution. Third, we carry out a double resummation involving both subdominant and sub-subdominant transseries to achieve the two-dimensional (in terms of the arbitrary constants) uniform approximation that allows the exploration of the behaviour of a two parameter set of solutions across wide regions of the independent variable. This is the first time all three effects have been studied jointly in the context of an asymptotic treatment of a nonlinear ordinary differential equation with a parameter. This paper provides an exponential asymptotic algorithm for attacking such problems when they occur. The availability of explicit results would depend on the individual equation under study.
\end{abstract}

AMS classification scheme numbers: 30E15, 34E05, 34E13, 34E15, 34E20, 34M35, 34M40, 34M45, 34M60

Submitted to: Nonlinearity

\section{Introduction}

The original purpose of this paper was to illustrate the effect of the the higher order Stokes phenomenon (HOS) [1], [2], on the asymptotic approximation of heteroclinic solutions of nonlinear ordinary differential equations (ode). This we certainly achieve, but in so doing we discover two further points of significant interest: a novel numerical approach to seeding and tracking specific solutions of nonlinear equations that are 
exponentially sensitive to initial data and a resummation scheme for exponentialasymptotic contributions to provide a doubly uniform approximation. For the case studied both these results allow for a quantitative and asymptotic exploration of the full possible set of solutions.

Having derived a novel exponential-asymptotic approximation, it is often expedient to check it against numerical solutions of an equation. For initial value problems, it is necessary to seed the differential equation with the appropriate initial data, and track the correct solution throughout the region of interest. In situations where only one subdominant exponential is present it often suffices to use an optimally-truncated asymptotic approximation to the solution and its derivatives to provide a sufficiently accurate starting point to track the relevant solution.

It is well known that a sum of asymptotic expansions may change in form as active Stokes curves are crossed in the space of parameters on which the coefficients depend. (see for example [3], [2]). Less well known is that a HOS may switch off the activity of a Stokes curve at a regular point in parameter space, ([4], [5], [6], [2], [1], [7]).

A HOS may occur whenever there is a sum of at least three series, each with a different exponential prefactor, $\exp \left(-f_{0} / \varepsilon\right), \exp \left(-f_{1} / \varepsilon\right), \exp \left(-f_{2} / \varepsilon\right)$, say, are present in a sum of asymptotic expansions. Away from turning points, the $f_{j}$ will all be different, and so there is an ordering in the $\Re\left(f_{j} / \varepsilon\right)$ : one asymptotic expansion is said to be dominant, one is sub dominant and the third is sub-subdominant. (For the reader who wishes to know more about the HOS at this stage, a brief guide is given, with references in section 6 below.)

Hence the occurrence of a HOS necessitates the tracking of sub-subdominant terms. Techniques illustrated in [2], [7] and [1] show that, in principle, it is possible to identify and follow such terms asymptotically. However a novel approach is required to seed and track this numerically.

Whilst it may be easy to pose arbitrary initial data and find an associated numerical solution, our requirement is to follow a specific numerical solution that has exponentially sub-subdominant terms present that arise from a specific choice of initial data. As such the initial data has to be of sufficient precision to allow the numerical solver to resolve the required solution. In the absence of closed form solutions, the numerical tracking of such solutions to verify the asymptotics is a longstanding challenge.

We do not claim a general solution to the problem of solution-tracking for systems which are exponentially sensitive to initial data. However we shall illustrate how some such problems might be attacked through the use of hyperasymptotic expansions ([8], [9], [10], [11], [12], [13], [14], [15]). Specifically, in certain circumstances, a hyperasymptotic asymptotic expansion can be used to provide better-than-exponential accuracy in the initial conditions, so allowing a numerical solver to track over an extended range an individual (shock) solution that is not captured by an attractor.

As hinted above, the presence of more than two exponential contributions also introduces novel effects for asymptotic approximations. Specifically, in the context of WKB-type solutions of higher order ordinary differential equations it has been known 
[4] that when more than two possible asymptotic behaviours are present, so-called "new Stokes lines" [5], [6], must be introduced to fully describe the analytic continuation.

In [1], [2] the concept of the higher order Stokes phenomenon (HOS) was introduced to explain the presence of these "new-Stokes line", demonstrating how the activity of Stokes lines in asymptotic expansions can be shown to change at regular points as they encounter higher order Stokes lines.

This effect, more subtle than the Stokes phenomenon, nevertheless has profound consequences that include changes in the asymptotic late time behaviour of solutions of partial differential equations due to the growth of initially exponentially subsubdominant terms, and in the absence of its consideration, the drawing of incorrect conclusions as to the existence of Stokes lines or singularities in the asymptotics as one traverses a space of control parameters. In [16] the effect of the higher order Stokes phenomenon in an inhomogeneous second order linear ordinary differential equation was illustrated. In [7] we showed in the context of Burgers equation how the presence of a higher order Stokes phenomenon prevents the presence of singularities in uniform asymptotic approximations of the solution after the formation of a nonlinear shock.

Here we aim to illustrate the effect of the HOS on nonlinear ordinary differential equations that exhibit "shock-type" heteroclinic behaviour. We shall confirm that, as with Burgers, the presence of a HOS is required to prevent singular behaviour of the asymptotic solutions in the vicinity of the "shock" transition between asymptotic behaviours.

The incorporation of a single exponentially small term by resumming it into a multiple-scales approach is a useful way of extending the range of validity of the solution as the dominance of the terms change, especially in nonlinear contexts [7], [17], [15]. However such uniform approaches are relatively unexplored when a third, initially sub-subdominant exponential, is also present. We shall show how to resum the transseries solutions to obtain a doubly-uniform asymptotic expression that captures all possible behaviours for any given initial data. We believe this to be the first example of a double resummation of transseries that contains two subdominant exponentials to obtain a multiple-scales approximation, valid through regions where a sub-subdominant exponential can grow to dominate.

We shall study the above in the context of the nonlinear ordinary differential equation satisfied by $y(z ; \varepsilon)$ where

$$
\varepsilon^{2} y^{\prime \prime}-\alpha \varepsilon y y^{\prime}+F(y, z)=0, \quad \alpha>0, \quad \varepsilon<<1 .
$$

Differentiation is with respect to the complex $z$-variable. We shall assume that $F$ is a polynomial in $y$

$$
F(y, z)=\sum_{q=0}^{Q} g_{q}(z) y^{q}
$$

where $Q$ is a positive integer and the $g_{q}$ are analytic in $z$.

This is by no means the first time that an exponential asymptotic approach ([18], [19], [20], [6]) - or even a hyperasymptotic one ([14], [15])- has been applied to nonlinear 
ordinary differential equations, but we believe this to be the first time a multiple resummation has been used in conjunction with the higher order Stokes phenomenon to uniformly track numerical solutions seeded by hyperasymptotic initial data.

The layout of the paper is as follows. In section 2 we extract the general features of transseries solutions of differential equations in class (1). In 3 we calculate terms in the expansion for a specific example and show how the leading order of the transseries be doubly resummed for the case in question. This leads to a two parameter solution that can be used to satisfy initial data. We derive the subdominant asymptotics in section 4. In order to identify the activity of the Stokes lines we derive the subsubdominant asymptotics in section 5 . In 6 we can then subsequently identify the location of turning points, Stokes curves and higher order Stokes curves. Hence we can make conjectures about the activity of the Stokes curves as they cross the higher order Stokes curves and the presence of a virtual turning point. It is also necessary to calculate the sub-subdominant terms, because for $z \rightarrow-\infty$, these terms grow to become the dominant solution, and so determine the nature of the hetero/homoclinic connection. In 7 we demonstrate how a hyperasymptotic approximation to the initial data can be used to lock on to a specific solution over an extended interval. In section 8 we verify the activity of the Stokes curves against numerical computations seeded with hyperasymptotic accuracy. Finally all these threads are drawn together in section 9 where the accuracy over an extended range of $z$ of the double resummation is numerically verified as a function of the underlying two free parameters, including its use to locate poles near to the virtual turning point where the solution undergoes a rapid change. The paper then concludes in section 10. Appendix A summarises the hyperasymptotic results that are used.

\section{General features of the formal solutions}

The general strategy starts by posing a template for the dominant formal solution $\hat{y}_{0}(z ; \varepsilon)$ of (1) as

$$
\hat{y}_{0}(z ; \varepsilon)=\sum_{s=0}^{\infty} a_{s}(z) \varepsilon^{s} .
$$

Substitution into (1) and balancing at leading order in $\varepsilon$ gives

$$
F\left(a_{0}(z), z\right)=0 .
$$

When $F$ is a nonlinear function of $a_{0}$ this will lead to a number of possible solutions. This contrasts with the nonlinear cases in [19] where $a_{0}$ is, a priori, uniquely determined.

A dominant formal solution of type (3) masks the possible existence of an infinite number of subdominant contributions called a transseries [20], [15]. These transseries involve small exponentials of the form $\exp (-f(z) / \varepsilon)$. Below we show that associated with these transseries are two degrees of freedom in that half plane, which in fact allows for specification of arbitrary initial data, where we also discuss the relationship between all possible choices of $a_{0}(z)$. 
In this paper we will often switch from formal series $\hat{y}_{j}(z ; \varepsilon)$ to actually functions $y_{j}(z ; \varepsilon)$ with the property $y_{j}(z ; \varepsilon) \sim \hat{y}_{j}(z ; \varepsilon)$ as $\varepsilon \rightarrow 0$. The function $y_{j}(z ; \varepsilon)$ will be the Borel-Laplace transform of $\hat{y}_{j}(z ; \varepsilon)$. For this definition and more details see [20].

For a given choice of $a_{0}(z)$, let us now investigate the presence of exponentially small terms and substitute a formal solution of the form

$$
y(z ; \varepsilon) \sim \hat{y}_{0}(z ; \varepsilon)+\sum_{j} \mathrm{e}^{-f_{j}(z) / \varepsilon} \hat{y}_{j}(z ; \varepsilon),
$$

for a finite set of labels $j$ that can be determined as follows. (Note that, it will be convenient to regard $y_{0}(z ; \varepsilon)$ as possessing a unit prefactor $\mathrm{e}^{-f_{0}(z) / \varepsilon}$ with $f_{0}(z) \equiv 0$.) Other types of asymptotic solutions may be possible, but we confine ourselves here to those which may be represented in the transseries for that starts with the form (5).

Substitution of (5) into (1) and balancing at $\mathcal{O}\left(\exp \left(-f_{j}(z) / \varepsilon\right)\right)$, we find that the $y_{j}(z ; \varepsilon)$ corresponding to formal series $\hat{y}_{j}(z ; \varepsilon)$ satisfy

$\varepsilon^{2} y_{j}^{\prime \prime}-\varepsilon\left(2 f_{j}+\alpha y_{0}\right) y_{j}^{\prime}+\left(f_{j}^{\prime 2}+\alpha y_{0} f_{j}^{\prime}+F_{y}\left(y_{0}, z\right)-\varepsilon\left(f_{j}^{\prime \prime}+\alpha y_{0}^{\prime}\right)\right) y_{j}=0$,

and from the $\mathcal{O}(1)$ terms in this equation we obtain

$$
f_{j}^{\prime 2}+\alpha a_{0} f_{j}^{\prime}+F_{y}\left(a_{0}, z\right)=0,
$$

(where the prime denotes differentiation with respect to $z$ and the subscript $y$ denote differentiation with respect to the first position in $F$ ). Thus there are two further possible $f_{j}(z)$ given by

$$
f_{j}^{\prime}=\frac{-\alpha a_{0}+(-1)^{j} \sqrt{\alpha^{2} a_{0}^{2}-4 F_{y}\left(a_{0}, z\right)}}{2}, \quad j=1,2 .
$$

For each non constant $a_{0}(z)$ for which $F_{y}\left(a_{0}, z\right) \not \equiv 0$, there is thus a basis of three independent $f_{j}^{\prime}$ for this second order equation, $j=0,1,2$.

Since the $f_{j}^{\prime}(z)$ satisfy (7) it follows that (6) has formal solutions of the form

$$
\hat{y}_{j}(z ; \varepsilon)=\sum_{s=0}^{\infty} b_{j, s}(z) \varepsilon^{s} .
$$

Note that so far the $f_{j}(z)$ are determined up to a constant, and since (6) is a linear homogeneous equation, the corresponding $y_{j}(z ; \varepsilon)$ are determined up to an arbitrary factor $C_{j}(\varepsilon)$ that can depend on $\varepsilon$. With this information we write

$$
y(z ; \varepsilon) \sim \hat{y}_{0}(z ; \varepsilon)+\sum_{j=1}^{2} C_{j}(\varepsilon) \mathrm{e}^{-f_{j}(z) / \varepsilon} \hat{y}_{j}(z ; \varepsilon) .
$$

A Stokes phenomenon may take place when we cross a so-called Stokes curve (see for example [2]), defined, for $\varepsilon>0$ by

$$
\Im\left(f_{i}(z)-f_{j}(z)\right)=0, \quad i \neq j,
$$

which emanates from a turning point $z_{t p}$. 
Crossing an active Stokes curve will switch on one of the exponentially small terms. In the case that we switch on the first small exponential we have as $\varepsilon \rightarrow 0^{+}$ $y(z ; \varepsilon) \sim \begin{cases}\hat{y}_{0}(z ; \varepsilon)+C_{1}(\varepsilon) e^{-f_{1}(z) / \varepsilon} \hat{y}_{1}(z ; \varepsilon) & \text { on one side of the Stokes curve, } \\ \hat{y}_{0}(z ; \varepsilon)+\left(C_{1}(\varepsilon)+K_{1}(\varepsilon)\right) e^{-f_{1}(z) / \varepsilon} \hat{y}_{1}(z ; \varepsilon) & \text { on the other side, }\end{cases}$

where

$$
K_{j}(\varepsilon) \sim \sum_{k=0}^{\infty} K_{j, k} \varepsilon^{k-\mu_{j}}
$$

are the so-called Stokes multipliers. Hence, the only unknowns in (12) are $\mu_{j}$ and the Stokes multipliers $K_{j, k}$. The $f_{j}(z)$ are so far known up to an only up to integration constant, see (8). However, to determine the location of the Stokes curves and to derive the exponentially small terms we must determine these constants.

We achieve a unique determination of the $f_{j}(z)$ in the analysis below by realising that the Stokes phenomenon is directly connected to the resurgence property that exists for nonlinear odes ([14], [15]):

$$
a_{n}(z) \sim \sum_{j=1}^{2} \sum_{k=0}^{\infty} \frac{K_{j, k}}{2 \pi i} \sum_{s=0}^{\infty} \frac{b_{j, s}(z) \Gamma\left(n-s-k+\mu_{j}\right)}{\left(f_{j}(z)\right)^{n-s-k+\mu_{j}}},
$$

as $n \rightarrow \infty$. Note that exactly the same $K_{j, k}$ and $\mu_{j}$ appear. We illustrate in this paper that often it is possible to determine the $\mu_{j}$ from (14), and at the moment that we know $\mu_{j}$ (14) can also be used to compute (at least numerically) as many of the Stokes multipliers $K_{j, k}$ as required. After the Stokes multipliers have been computed it is more convenient to introduce

$\beta_{j, s}(z)=\sum_{k=0}^{s} K_{j, k} b_{j, s-k}(z), \quad$ and redefine $\quad \hat{y}_{j}(z ; \varepsilon)=\sum_{s=0}^{\infty} \beta_{j, s}(z) \varepsilon^{s-\mu_{j}}$,

then (14) becomes

$$
a_{n}(z) \sim \sum_{j=1}^{2} \frac{1}{2 \pi i} \sum_{s=0}^{\infty} \frac{\beta_{j, s}(z) \Gamma\left(n-s+\mu_{j}\right)}{\left(f_{j}(z)\right)^{n-s+\mu_{j}}},
$$

as $n \rightarrow \infty$, and the Stokes phenomenon of switching on the first small exponential is now of the form

$y(z ; \varepsilon) \sim\left\{\begin{array}{l}\hat{y}_{0}(z ; \varepsilon)+C_{1}(\varepsilon) e^{-f_{1}(z) / \varepsilon} \hat{y}_{1}(z ; \varepsilon) \quad \text { on one side of the Stokes curve, } \\ \hat{y}_{0}(z ; \varepsilon)+\left(C_{1}(\varepsilon) \pm 1\right) e^{-f_{1}(z) / \varepsilon} \hat{y}_{1}(z ; \varepsilon) \text { on the other side, }\end{array}\right.$

the \pm depending on the direction of the crossing.

Note that so far we have not defined the turning points. In fact it is not easy to give a precise definition. At a turning point the asymptotic expansions break down. For example, if the $a_{n}(z)$ have a singularity at $z=z_{t p}$, then for one of the $f_{j}, j=1,2$, we will have that $f\left(z_{t p}\right)=0$, and note that in this case the singular behaviour is incorporated in asymptotic approximation (16). Usually, it is not difficult to determine the singularities 
of the $a_{n}(z)$, and the requirement $f\left(z_{t p}\right)=0$ fixes the integration constant in (8). However, not all turning points can be determined in this way. We illustrate that singular behaviour of coefficients $b_{j, s}(z)$ can also be connected to a turning point, and in our example this will be used to determine the integration constant of $f_{2}(z)$ and the second turning point.

Since (1) is nonlinear, substituting in the form (10) and balancing at each exponential order shows that the the basic exponential scales in (10) generate an infinite array of subdominant exponential contributions of the form $C_{1}^{m}(\varepsilon) C_{2}^{n}(\varepsilon) e^{-\left(m f_{1}(z)+n f_{2}(z)\right) / \varepsilon}$. Note that the only free parameters will be $C_{1}(\varepsilon)$ and $C_{2}(\varepsilon)$, and when we cross, for example, the Stokes curve that switches on the first small exponential, then in these double series all the $C_{1}(\varepsilon)$ are replaced by $C_{1}(\varepsilon) \pm 1$, compare (17). This is the Stokes phenomenon for nonlinear differential equations. More details are given in [14].

Given this, the transseries (10) actually takes the more specific form

$y(z ; \varepsilon)=\sum_{m=0}^{\infty} \sum_{n=0}^{\infty} C_{1}^{m}(\varepsilon) C_{2}^{n}(\varepsilon) A_{m, n}(z) \mathrm{e}^{-\left(m f_{1}(z)+n f_{2}(z)\right) / \varepsilon}+O(\varepsilon), \quad$ as $\varepsilon \rightarrow 0^{+}$,

with $A_{0,0}(z)=a_{0}(z), A_{1,0}(z)=b_{1,0}(z)$ and $A_{0,1}(z)=b_{2,0}(z)$.

Initially, we can choose to start in regions where these terms will be exponentiallysmall, but when we travel in the complex $z$-plane, these terms may grow in size to become $\mathcal{O}(1)$ near a so-called anti-Stokes line, where they cannot be neglected, [7]. That is why they are retained even when the error estimate in $(35)$ is $\mathcal{O}(\varepsilon)$. Wherever the latter estimate occurs, it refers to the presence of only the leading order terms in each of the component exponentially-prefactored series.

Substituting (18) into (1) and balancing at each exponential order, we obtain

$$
\begin{array}{r}
\left(m f_{1}^{\prime}+n f_{2}^{\prime}\right)^{2} A_{m, n}+\alpha \sum_{\substack{m_{1}+m_{2}=m \\
n_{1}+n_{2}=n}}\left(m_{1} f_{1}^{\prime}+n_{2} f_{2}^{\prime}\right) A_{m_{1}, n_{1}} A_{m_{2}, n_{2}} \\
+\sum_{q=1}^{Q} g_{q}(z) \sum_{\substack{m_{1}+\cdots+m_{q}=m \\
n_{1}+\cdots+n_{q}=n}} \prod_{s=1}^{q} A_{m_{s}, n_{s}}=0 .
\end{array}
$$

In general this recurrence relation cannot be solved analytically, but in many special examples one might be lucky, like in the example in this paper. However, since it is a relatively simple recurrence relation, as many of the $A_{m, n}(z)$ can be computed numerically as desired. They contain information on the behaviour near the anti-Stokes lines

$$
\Re\left(f_{i}(z)-f_{j}(z)\right)=0, \quad i \neq j .
$$

It should be possible to obtain information about singularities of the solutions of an individual ode under study near the anti-Stokes line, either individually from the $A_{m, n}$ or by a numerical, if not analytical, resummation, as we show below. 


\section{Calculations for specific example}

In order to make further concrete progress we now consider a specific example. We stress that in what follows, the general algorithm of attack would be similar for different equations in this class. However the degree to which one might achieve explicit results will depend, as always in asymptotics, on the solvability of the intermediate steps.

We shall henceforth consider the special case with $\alpha=3$ and

$$
\begin{aligned}
& g_{0}(z)=-i\left(\frac{2}{27} z^{3}+\frac{2}{3} z\right), g_{1}(z)=\frac{1}{3} z^{2}-1, \\
& g_{2}(z)=0, g_{3}(z)=1, g_{i}(z)=0, i \geq 4
\end{aligned}
$$

that is, our main equation reads

$\varepsilon^{2} y^{\prime \prime}(z ; \varepsilon)-3 \varepsilon y(z ; \varepsilon) y^{\prime}(z ; \varepsilon)+\left(y^{3}(z ; \varepsilon)+\left(\frac{1}{3} z^{2}-1\right) y(z ; \varepsilon)\right)=i\left(\frac{2}{27} z^{3}+\frac{2}{3} z\right)$.

Although the resulting equation looks somewhat complicated, it has been chosen for the simple geometric structure of its Stokes and higher order Stokes curves (straight lines and a circle, respectively), to facilitate the explanations (see figure 1 below). The form of the equation introduces notable pedagogical features that nevertheless may be encountered elsewhere in nonlinear (and other) ordinary differential equations.

The leading order equation for $a_{0}(z)$ becomes

$$
a_{0}^{3}(z)+\left(\frac{1}{3} z^{2}-1\right) a_{0}(z)=i\left(\frac{2}{27} z^{3}+\frac{2}{3} z\right)
$$

with three possible solutions

$$
a_{0}(z)=\left\{\begin{array}{l}
\alpha_{ \pm}(z)=\frac{1}{3} i z \pm 1 \\
\alpha_{0}(z)=-\frac{2}{3} i z
\end{array}\right.
$$

In the analysis below it will follow that the imaginary axis is an anti-Stokes line for the dominant Poincaré series $\hat{y}_{0}(x, \varepsilon)$. Our initial analysis will focus on $\Re(z)>0$. The absence of any free parameters in (24) suggests that there is only a zero dimensional set of initial data which may be satisfied by any of the three specific solutions of (22). Recall that two free constants appear in (10).

We now turn to the procedure for selecting the appropriate $a_{0}(z)$. When $a_{0}(z)=$ $\alpha_{-}(z)$ we obtain from $(8)$ that $f_{1}^{\prime}(z)=1-i z, f_{2}^{\prime}(z)=2$, for $a_{0}(z)=\alpha_{+}(z)$ we have $f_{1}^{\prime}(z)=-1-i z, f_{2}^{\prime}(z)=-2$, and for the choice $a_{0}(z)=\alpha_{0}(z)$ we have $f_{1}^{\prime}(z)=i z+1$, $f_{2}^{\prime}(z)=i z-1$. Hence, only for the first choice are the corresponding $f_{j}(z)$ both increasing along the real axis. Hence, only in this case the two possible small exponentials will remain small as $z$ increases. This solution is the main attractor as $z$ increases. We will take $a_{0}(z)=\alpha_{-}(z)$ :

$$
a_{0}(z)=\frac{1}{3} i z-1 \text {. }
$$

If we were to chose either of the other two non-attracting solutions $\alpha_{0}(z)$ or $\alpha_{+}(z)$ in the presence of their transseries, then as $z \rightarrow+\infty$, terms involving at least one of the exponential prefactors $\exp \left(-f_{1}(z) / \varepsilon\right)$ or $\exp \left(-f_{2}(z) / \varepsilon\right)$ would grow exponentially in size to dominate the $a_{0}(z)$. On the one hand, this would violate the assumption 
that the dominant term is the chosen $a_{0}(z)$, but on the other, as we show below, from the resummed transseries in this example the growth of these terms would combine to generate the attracting solution $\alpha_{-}(z)$.

For $s \geqslant 0$ (with $a_{-1} \equiv 0$ ), balancing at powers of $u$, we then have

$$
\begin{gathered}
2 i(z+i) a_{s+1}(z)=a_{s-1}^{\prime \prime}(z)-3 \sum_{p=0}^{s} a_{p}^{\prime}(z) a_{s-p}(z)+a_{0}(z) \sum_{p=1}^{s} a_{p}(z) a_{s-p+1}(z) \\
+\sum_{p=1}^{s} \sum_{q=0}^{p} a_{q}(z) a_{p-q}(z) a_{s-p+1}(z) .
\end{gathered}
$$

We note from the form of (26) that for $s \geqslant 1$ the coefficient $a_{s}(z)$ is a rational function with a single singularity, a pole of order $2 s-1$ at $z=-i$. It follows that $z=-i$ is a turning point for the differential equation. The singularity structure of these terms provides the skeletal structure for a significant part of the analysis that follows.

For our special example (6) now reads

$$
\begin{aligned}
\varepsilon^{2} y_{j}^{\prime \prime}(z ; \varepsilon)-\varepsilon\left(3 y_{0}(z ; \varepsilon)\right. & \left.+2 f_{j}^{\prime}(z)\right) y_{j}^{\prime}(z ; \varepsilon)+\left[f_{j}^{\prime 2}(z)+3 y_{0}(z ; \varepsilon) f_{j}^{\prime}(z)\right. \\
& \left.+\frac{1}{3} z^{2}-1+3 y_{0}^{2}(z ; \varepsilon)-\varepsilon\left(f_{j}^{\prime \prime}(z)+3 y_{0}^{\prime}(z ; \varepsilon)\right)\right] y_{j}(z ; \varepsilon)=0,
\end{aligned}
$$

and (7) becomes

$$
f_{j}^{\prime 2}(z)+(i z-3) f_{j}^{\prime}(z)-2 i z+2=0,
$$

with two solutions

$$
f_{1}(z)=-\frac{1}{2} i(z+i)^{2}+k_{1}, \quad \text { and } \quad f_{2}(z)=2 z+k_{2} .
$$

where the constants $k_{1}$ and $k_{2}$ are arbitrary at the moment. With these choices for $f_{j}(z)$ we obtain from $(27)$ that $y_{1}(z ; \varepsilon) \sim C_{1}(\varepsilon) b_{1,0}(z)$ and $y_{2}(z ; \varepsilon) \sim C_{2}(\varepsilon) b_{2,0}(z)$, where, since (27) is a linear equation, $C_{j}(\varepsilon), j=1,2$, are arbitrary functions of $\varepsilon$, and where

$i(i-z) b_{1,0}^{\prime}(z)+\frac{z^{2}+9}{6(z+i)} b_{1,0}(z)=0, \quad$ and $i(i-z) b_{2,0}^{\prime}(z)+\frac{z^{2}+3}{3(z+i)} b_{2,0}(z)=0$.

Since the $C_{j}(\varepsilon)$ are arbitrary, (30) can be solved without loss of generality to give

$$
b_{1,0}(z)=\mathrm{e}^{-i(z+i) / 6}\left(\frac{i+z}{i-z}\right)^{2 / 3}, \text { and } b_{2,0}(z)=\mathrm{e}^{-i z / 3}\left(\frac{i+z}{i-z}\right)^{1 / 3} .
$$

Although it seems that the $C_{j}(\varepsilon)$ are arbitrary functions of $\varepsilon$, we note that for (10) to be a valid ansatz (in the sense that it is composed of a decreasing sequence of exponentials) we insist that $\mathrm{e}^{-f_{j}(z) / \varepsilon} y_{j}(z ; \varepsilon)$ are initially exponentially small. In turn, from the form of $y_{1}, y_{2}$ above, this imposes the growth condition that $C_{j}(\varepsilon)=\mathcal{O}\left(\mathrm{e}^{k / \varepsilon}\right)$, as $\varepsilon \rightarrow 0^{+}$, for some positive constant $k$. Consequently, as the $C_{j}(\varepsilon)$ can grow like an exponential at most linearly in $1 / \varepsilon$, we can therefore take $k_{1}=k_{2}=0$ in (29), without loss of generality. For the transseries ansatz the choice of $k_{j}$ are not crucial. Sometimes it is convenient use initial data to make a choice (see section 3.1 below). However if one wants to travel around the $z$-plane it is necessary to incorporate changes in the asymptotic representation arising from the Stokes phenomenon. In that case it is vital determine the 
$k_{j}$ in a way that is consistent with the singularity structure of the expansion coefficients arising from the turning points. We demonstrate how to do this below, where in fact the $k_{j}=0$ anyway.

Substituting (18) into (22) and balancing at each exponential order, the recurrence relation (19) for the leading orders of the terms in the transseries becomes

$$
\begin{aligned}
\left(\left(m f_{1}^{\prime}+n f_{2}^{\prime}\right)^{2}+\frac{1}{3} z^{2}-1\right) A_{m, n} & +3 \sum_{\substack{m_{1}+m_{2}=m \\
n_{1}+n_{2}=n}}\left(m_{1} f_{1}^{\prime}+n_{1} f_{2}^{\prime}\right) A_{m_{1}, n_{1}} A_{m_{2}, n_{2}} \\
& +\sum_{\substack{m_{1}+m_{2}+m_{3}=m \\
n_{1}+n_{2}+n_{3}=n}} A_{m_{1}, n_{1}} A_{m_{2}, n_{2}} A_{m_{3}, n_{3}}=0 .
\end{aligned}
$$

The general solution of (32) is

$A_{m, n}(z)=-\left(\frac{\Gamma(n+m) f_{1}^{\prime}(z)}{\Gamma(m) \Gamma(n+1)}+\frac{\Gamma(n+m) f_{2}^{\prime}(z)}{\Gamma(m+1) \Gamma(n)}\right)\left(\frac{-b_{1,0}(z)}{f_{1}^{\prime}(z)}\right)^{m}\left(\frac{-b_{2,0}(z)}{f_{2}^{\prime}(z)}\right)^{n}$,

$(m, n) \neq(0,0)$, with the understanding that when $n=0$ or $m=0$ we have, respectively

$$
A_{m, 0}(z)=\frac{\left(b_{1,0}(z)\right)^{m}}{\left(-f_{1}^{\prime}(z)\right)^{m-1}} \text { and } A_{0, n}(z)=\frac{\left(b_{2,0}(z)\right)^{n}}{\left(-f_{2}^{\prime}(z)\right)^{n-1}} .
$$

Substituting these expressions into the right-hand side of (18), in regions where $\Re(z)$ is sufficiently large, we observe that the double sum is of hypergeometric type (see [21]) and we can actually perform a double resummation to obtain

$y(z ; \varepsilon)=\alpha_{-}(z)+\frac{C_{1}(\varepsilon) b_{1,0}(z) \mathrm{e}^{-f_{1}(z) / \varepsilon}+C_{2}(\varepsilon) b_{2,0}(z) \mathrm{e}^{-f_{2}(z) / \varepsilon}}{1+C_{1}(\varepsilon) b_{1,0}(z) \mathrm{e}^{-f_{1}(z) / \varepsilon} / f_{1}^{\prime}(z)+C_{2}(\varepsilon) b_{2,0}(z) \mathrm{e}^{-f_{2}(z) / \varepsilon} / f_{2}^{\prime}(z)}+O(\varepsilon),(35$

as $\varepsilon \rightarrow 0^{+}$.

Although we started with the choice of $a_{0}(z)=\alpha_{-}(z)$, the resummation (35) uniformly covers and smoothly interpolates between all three of the possible algebraically dominant behaviours for $a_{0}(z)$ :

$y(z) \sim \begin{cases}\alpha_{-}(z), & C_{1}(\varepsilon) \mathrm{e}^{-f_{1}(z) / \varepsilon} \ll 1 \& C_{2}(\varepsilon) \mathrm{e}^{-f_{2}(z) / \varepsilon} \ll 1, \\ \alpha_{-}(z)+f_{1}^{\prime}(z)=\alpha_{0}(z), & C_{1}(\varepsilon) \mathrm{e}^{-f_{1}(z) / \varepsilon} \gg 1 \& C_{1}(\varepsilon) \mathrm{e}^{-f_{1}(z) / \varepsilon} \gg C_{2}(\varepsilon) \mathrm{e}^{-f_{2}(z) / \varepsilon}, \\ \alpha_{-}(z)+f_{2}^{\prime}(z)=\alpha_{+}(z), & C_{2}(\varepsilon) \mathrm{e}^{-f_{2}(z) / \varepsilon} \gg 1 \& C_{1}(\varepsilon) \mathrm{e}^{-f_{1}(z) / \varepsilon} \ll C_{2}(\varepsilon) \mathrm{e}^{-f_{2}(z) / \varepsilon} .\end{cases}$

We thus see from the double resummation of the transseries that the arbitrary $C_{j}(\varepsilon)$ can in fact be used to generate asymptotic approximations to the required two-dimensional set of possible solutions, for any given point $z_{0}$ at which initial data is given, covering all possible dominant behaviours.

Additionally, we shall show in the second half of section 9 how the double resummation (35) can be used to approximate the positions of the first array of poles encountered by a solution $y(z ; \varepsilon)$ as it enters from a pole-free region in the complex $z$-plane.

It is important to realise that the ability to resum the transseries terms $A_{m, n}(z)$ analytically and the subsequent form will depend on the specific differential equation under study. However, for equations in the class (1) with the form of $F$ outlined, it 
should be possible to obtain a recurrence relation between the $A_{m, n}(z)$ analogous to the form (32). The solutions of such recurrence relations can be expressed in terms of an expansion in powers of $m$ and $n$ in (33), with coefficients that can be determined, at least numerically from the $A_{m, n}(z)$ recurrence relation, provided that the $b_{1,0}$ and $b_{2,0}$ and $C_{1}(\varepsilon)$ and $C_{2}(\varepsilon)$ for the specific solution are known. Hence it is, in principle, possible to obtain a numerical resummation of the transseries for the differential equation under study, up to the order of numerical truncation that the user chooses. An alternative approach is to take an appropriate double $Z$-transform of (32) to obtain an equation for doubly-resummed transseries, that might be solvable analytically. For details see [22].

Henceforth, we shall work with specific values of $\varepsilon$ and so the $C_{j}$ can be treated as constants, which we shall find.

To our knowledge, this is the first time double transseries resummation has been examined in detail.

\subsection{Determining solutions via initial data}

We now discuss issue of relating the transseries solutions to a given set of initial data.

Our main differential equation is of second order. Hence, given a fixed small $\varepsilon$ and initial data $y\left(z_{0} ; \varepsilon\right)=Y_{0}$ and $y^{\prime}\left(z_{0} ; \varepsilon\right)=Y_{1}$, where $z_{0}$ is a fixed real point, the solution of $(22)$ should be uniquely determined. It is unlikely that $\hat{y}_{0}\left(z_{0}, \varepsilon\right)$ is an asymptotic approximation to this solution, since the Poincaré series has no free constants. However, the right-hand side in (35), that is, the resummed transseries, has two degrees of freedom $C_{1}(\varepsilon)$ and $C_{2}(\varepsilon)$. We can use this at follows:

First fix the constants $k_{j}$ in $(29)$ via $f_{j}\left(z_{0}\right)=0$. Now take a real $z$ close $z_{0}$ such that $z>z_{0}$. Then $\Re\left(f_{j}(z)\right)>0$. Hence, the exponentials in the transseries are exponentially small. On the interval $\left(z_{0}, z\right)$ the transseries can be resummed, and its sum is the righthand side in (35). Since (35) has two free constants we can match it to the initial data at $z_{0}$. With our choice $f_{j}\left(z_{0}\right)=0$ we guarantee that the exponentials are $\mathcal{O}(1)$ at $z_{0}$, and in the case that the $Y_{0}$ and $Y_{1}$ are bounded, the free parameters $C_{1}$ and $C_{2}$ will also be bounded.

\section{Calculation of subdominant series}

We now turn to the problem of calculating the subdominant terms in the transseries expansion. We will need these to verify the activity of the Stokes lines. To that end we seek to calculate the $\beta_{1, s}(z)$ terms in (16).

Taking $f_{j}(z)=f_{1}(z)$ in $(27)$ gives us the differential equation

$$
\begin{aligned}
& \varepsilon^{2} y_{1}^{\prime \prime}(z ; \varepsilon)-\varepsilon\left(3 y_{0}(z ; \varepsilon)+2-2 i z\right) y_{1}^{\prime}(z ; \varepsilon) \\
& +\left(\left(3(1-i z) y_{0}(z ; u)+3 y_{0}^{2}(z ; \varepsilon)-\frac{2}{3} z^{2}-2 i z\right)+\varepsilon\left(i-3 y_{0}^{\prime}(z ; \varepsilon)\right)\right) y_{1}(z ; \varepsilon)=0,
\end{aligned}
$$

with formal solution $\hat{y}_{1}(z ; \varepsilon)$ (see $\left.(9)\right)$, where, from $(31)$,

$$
b_{1,0}(z)=\mathrm{e}^{-i(z+i) / 6}\left(\frac{i+z}{i-z}\right)^{2 / 3}
$$


and for $s \geq 0$,

$$
\begin{aligned}
& i(i-z) b_{1, s+1}^{\prime}(z)+\frac{z^{2}+9}{6(z+i)} b_{1, s+1}(z) \\
& =b_{1, s}^{\prime \prime}(z)-3 \sum_{p=0}^{s}\left(b_{1, p}^{\prime}(z) a_{s+1-p}(z)+b_{1, p}(z) a_{s+1-p}^{\prime}(z)\right) \\
& \quad-3 i(z+i) \sum_{p=0}^{s} b_{1, p}(z) a_{s+2-p}(z)+3 \sum_{p=0}^{s} \sum_{q=p}^{s+2} b_{1, p}(z) a_{q-p}(z) a_{s+2-q}(z) .
\end{aligned}
$$

Note that (37) is a linear homogeneous equation. Hence, multiplying $\hat{y}_{1}(z ; \varepsilon)$ by any arbitrary power series in powers of $\varepsilon$ will also give a formal solution. This is reflected in the fact that recurrence relation (39) requires an integration to determine each $b_{1, s}$, $(s \geq 1)$. Each $b_{1, s}$ will contain an arbitrary integration constant. The constants of the integration for the $b_{1, s}(z)$ in (39) are chosen as follows. We choose in the representation $b_{1, s}(z)=b_{1,0}(z) \tilde{b}_{1, s}(z)$ in the local expansion for $\tilde{b}_{1, s}(z)$ at $z=-i$, the constant term equal to zero. There is no deep reason for this choice, it just determines the $b_{1, s}(z)$ uniquely. The consequences are fully accounted for as follows.

When $|z|<1$, the $b_{1, s}(z)$ terms are connected to the growth of the coefficients $a_{n}(z)$ by:

$$
a_{n}(z) \sim \sum_{k=0}^{\infty} \frac{K_{1, k}}{2 \pi i} \sum_{s=0}^{\infty} \frac{b_{1, s}(z) \Gamma\left(n-s-k+\mu_{1}\right)}{\left(f_{1}(z)\right)^{n-s-k+\mu_{1}}},
$$

as $n \rightarrow \infty$. The $K_{1, k}$ are Stokes multipliers to be determined, their values are related to the choice of arbitrary constants of integration in (39). Different choices of contants will lead to compensating values of the Stokes multipliers $K_{1, k}$. The parameter $\mu_{1}$ is determined below.

Using (15) we reduce the double sum in (40) to a single sum

$$
a_{n}(z) \sim \frac{1}{2 \pi i} \sum_{s=0}^{\infty} \frac{\beta_{1, s}(z) \Gamma\left(n-s+\mu_{1}\right)}{\left(f_{1}(z)\right)^{n-s+\mu_{1}}},
$$

as $n \rightarrow \infty$.

We now demonstrate a general method to calculate the Stokes multipliers $K_{1, k}$.

Note that in (40), we can find both the $a_{n}(z)$ and $b_{1, s}(z)$ from recurrence relations (26) and (39) respectively. Hence the only unknowns are the Stokes multipliers $K_{1, k}$ and $\mu_{1}$ (which will be needed later). Numerical comparisons of the terms in (40) give

$$
K_{1,0}=\frac{2^{7 / 6} \mathrm{e}^{5 \pi i / 12} \sqrt{\pi}}{\Gamma\left(\frac{1}{3}\right)}, \quad \mu_{1}=-\frac{1}{6}, \quad K_{1,1}=\frac{19}{72} i K_{1,0} .
$$

We compute the other Stokes multipliers numerically via (40) by taking $z=0$, $n=115, \cdots, 120$, with 8 terms in the $k$-sum and 31 terms in the $s$ sum. (The larger the values of $n$ and $s$, the more accurate the results.) In this way we obtain 6 equations with the unknowns $K_{1,2}, \cdots, K_{1,7}$. This set of linear algebraic equations can be solved numerically by standard techniques. The result is:

$K_{1,2}=-0.1841242284 K_{1,0}, \quad K_{1,3}=-0.2194925801 i K_{1,0}, \quad K_{1,4}=0.4153980454 K_{1,0} \cdot(43)$ 
Using $K_{1,0}$ in the right-hand sides of these results makes the presentation a bit shorter. To show that the first part of (42) is correct, we study the coefficients $a_{s}(z)$ near $z=-i$ and write

$$
a_{s}(z)=\frac{A_{s}}{(z+i)^{2 s-1}}+\mathcal{O}\left((z+i)^{2-2 s}\right), \quad \text { as } z \rightarrow-i,
$$

where $A_{1}=\frac{1}{3}$. It follows from (26) that for $s \geqslant 2$ we have

$$
i A_{s}+(2 s-3) A_{s-1}+\sum_{p=1}^{s-1} A_{p} A_{s-p}=0 .
$$

Hence, the formal expansion

$$
\hat{v}(z)=\sum_{s=1}^{\infty} \frac{A_{s}}{z^{s}},
$$

is a solution of the Riccati equation

$$
-2 v^{\prime}(z)+\left(i-\frac{1}{z}\right) v(z)+v^{2}(z)-\frac{i}{3 z}=0 .
$$

It is not difficult to verify that the Borel-Laplace transform [13] of asymptotic expansion $\hat{v}(z)$ is

$$
v(z)=\frac{-2 \frac{d}{d z}\left(\sqrt{z} U\left(\frac{2}{3}, \frac{3}{2}, \frac{i z}{2}\right)\right)}{\sqrt{z} U\left(\frac{2}{3}, \frac{3}{2}, \frac{i z}{2}\right)},
$$

where $U(a, b, z)$ is the confluent hypergeometric $U$-function ([23] 113.2$)$. Since the Stokes constants for the functions in (48) are known (see (13.2.12) in [23]) it follows that

$$
A_{n} \sim \frac{2 \Gamma\left(n-\frac{1}{6}\right)}{\Gamma\left(\frac{1}{6}\right) \Gamma\left(\frac{2}{3}\right)(-i / 2)^{n-1}}, \quad \text { as } n \rightarrow \infty .
$$

Combining (40) with (44) and (49), it follows that the first two identities in (42) hold. Analytical verification of the higher order $K_{1, k}, k>1$ may also be possible.

We remark that using the techniques of exponential and hyper- asymptotics it is normally possible systematically to calculate numerical approximations to Stokes multipliers arising from differential equations as we have done in (43). In this case we are able to confirm the analytical form of $K_{1,0}$ due to the form of the original differential equation. Even if an analytical expression for $K_{1,0}$ could not be obtained, the above method (extended to 8 unknowns by including two more equations of the form (40) with $n=121,122)$ still provides consistent numerical values for all the Stokes constants.

\section{Calculation of sub-subdominant series}

A necessary condition for the existence of a higher order Stokes phenomenon (and so the ability of a Stokes line to end at a regular point in the plane) is the presence of three different exponential behaviours. In this context these translate to a dominant, subdominant and sub-subdominant contribution. In previous sections 
we have calculated the former two. In this section we calculate the latter, the subsubdominant coefficients $\beta_{2, s}(z)$ of $(65)$.

Their contribution to the asymptotics of solutions is sub-subdominant in regions of the complex $z$-plane when $\Re\left\{\left(u\left(f_{2}(z)-f_{1}(z)\right)\right\}>0\right.$ (where $\varepsilon>0, \Re(z)>0$ and $\Im(z)<1$ ) but will grow to dominate the asymptotics near $z=0$.

There are two ways in which the $\beta_{2, s}(z)$ series can appear in the asymptotics of a solution. First they may be switched on across a Stokes line by the $a_{s}(z)$ series (in this case the half line $z>1$ ). Secondly the $\beta_{1, s}(z)$ series may switch them on where $\left(f_{2}(z)-f_{1}(z)\right) / \varepsilon>0$, in this case line $(b)$ of figure 2 .

The $i-z$ in (38), and hence in $\beta_{1,0}(z)$ and (66), indicates that $z=i$ is a turning point: in (66) the right-hand side has a singularity at $z=i$ but the left-hand side is analytic at this point. One way to circumvent this is to require that the optimal number of terms in the $\beta_{1, s}(z)$ series has to be zero when $z=i$. Hence we need $f_{1}(i)=f_{2}(i)$, that is,

$$
f_{2}(z)=2 z
$$

Taking $f_{j}(z)=f_{2}(z)$ in $(27)$ gives us the differential equation

$$
\begin{aligned}
& \varepsilon^{2} y_{2}^{\prime \prime}(z ; \varepsilon)-\varepsilon\left(3 y_{0}(z ; \varepsilon)+4\right) y_{2}^{\prime}(z ; \varepsilon)+ \\
& \left(\left(6 y_{0}(z ; \varepsilon)+3 y_{0}^{2}(z ; \varepsilon)+\frac{1}{3} z^{2}+3\right)-3 \varepsilon y_{0}^{\prime}(z ; \varepsilon)\right) y_{2}(z ; \varepsilon)=0
\end{aligned}
$$

with formal solution $\hat{y}_{2}(z ; \varepsilon)$ (see $\left.(9)\right)$, where, from (31),

$$
b_{2,0}(z)=\mathrm{e}^{-i z / 3}\left(\frac{i+z}{i-z}\right)^{1 / 3}
$$

and for $s \geq 0$,

$$
\begin{aligned}
& i(z-i) b_{2, s+1}^{\prime}(z)-\frac{z^{2}+3}{3(z+i)} b_{2, s+1}(z) \\
& =b_{2, s}^{\prime \prime}(z)-3 \sum_{p=0}^{s}\left(b_{2, p}^{\prime} p(z) a_{s+1-p}(z)+b_{2, p}(z) a_{s+1-p}^{\prime}(z)\right) \\
& \quad+6 \sum_{p=0}^{s} b_{2, p}(z) a_{s+2-p}(z)+3 \sum_{p=0}^{s} \sum_{q=p}^{s+2} b_{2, p}(z) a_{q-p}(z) a_{s+2-q}(z) .
\end{aligned}
$$

For values of $z$ outside the cardioid in figure 1, these terms are connected to the growth of the coefficients $a_{n}(z)$ by the resurgence expansion:

$$
a_{n}(z) \sim \sum_{k=0}^{\infty} \frac{K_{2, k}}{2 \pi i} \sum_{s=0}^{\infty} \frac{b_{2, s}(z) \Gamma\left(n-s-k+\mu_{2}\right)}{\left(f_{2}(z)\right)^{n-s-k+\mu_{2}}},
$$

as $n \rightarrow \infty$. The $b_{1, s}(z)$ series in (14) is also present in this region but are asymptotically dominated by the $b_{2, s}(z)$ for large $|z|$.

The constants of the integration in (53) are chosen as follows: by writing $b_{2, s}(z)=$ $b_{2,0}(z) \tilde{b}_{2, s}(z)$ where

$$
\tilde{b}_{2, s}(z) \sim \sum_{p=-s}^{\infty} \frac{e_{p, s}}{z^{p}}, \quad z \rightarrow \infty
$$


and choosing the constant term $e_{0, s}$ to be equal to zero. Again, alternative choices for the constants of integration lead to compensating values of the Stokes multipliers. We remark that the choice $e_{0, s}=0$ leads to numerically stable calculations.

Once again, we know all the terms in (54) except for the Stokes multipliers $K_{2, k}$ and $\mu_{2}$. Numerical comparisons give $\mu_{2}=0$,

$$
K_{2,0}=2 \sqrt{3} \mathrm{e}^{5 \pi i / 6}, \quad K_{2,1}=\frac{i}{6} K_{2,0} .
$$

Using again (15) reduces the double sum in (54) to a single sum

$$
a_{n}(z) \sim \frac{1}{2 \pi i} \sum_{s=0}^{\infty} \frac{\beta_{2, s}(z) \Gamma(n-s)}{\left(f_{2}(z)\right)^{n-s}},
$$

as $n \rightarrow \infty$.

We compute the other Stokes multipliers $K_{2, k}$ numerically via (54) by taking $z=4+4 i, n=115, \cdots, 120$, with 8 terms in the $k$-sum and 31 terms in the $s$ sum. (Again the larger the values of $n$ and $s$, the greater the accuracy.) In this way we obtain 6 equations with the unknowns $K_{2,2}, \cdots, K_{2,7}$ and the result is:

$K_{2,2}=0.0231481481 K_{2,0}, \quad K_{2,3}=-0.0034722220 i K_{2,0}, \quad K_{2,4}=-0.0007160438 K_{2,0} \cdot(58)$

Using $K_{2,0}$ in the right-hand sides of these results again makes the presentation a bit shorter. In the absence of an analytical representation for $K_{2,0}$ the numerical values of the $K_{i, j}$ could be found by extending the number of equations in the numerical system based on (54) by two (e.g., $n=121,122)$ to calculate $K_{2,0}$ and $K_{2,1}$ numerically.

We can verify that $\mu_{2}=0$ and $K_{2,0}=2 \sqrt{3} \mathrm{e}^{5 \pi i / 6}$ analytically by considering the asymptotic expansion of the $b_{1, n}(z)$ in terms of the $b_{2, s}(z)$, as follows.

We first determine the Stokes multiplier of $f_{1}$ switching on $f_{2}$. The local behaviour of the coefficients $b_{1, n}(z)$ near $z=i$ follows from (38) and (39):

$b_{1, n}(z) \sim d_{n}(z-i)^{-2 n-2 / 3}, \quad$ as $z \rightarrow i, \quad$ where $2 i(n+1) d_{n+1}=\left(2 n+\frac{2}{3}\right)\left(2 n+\frac{5}{3}\right) d_{n}$.

Thus we have

$d_{0}=\mathrm{e}^{1 / 3}(-2 i)^{2 / 3}, d_{n}=\frac{\Gamma\left(n+\frac{1}{3}\right) \Gamma\left(n+\frac{5}{6}\right)}{\Gamma\left(\frac{1}{3}\right) \Gamma\left(\frac{5}{6}\right) n !}(-2 i)^{n} d_{0} \sim \frac{\mathrm{e}^{1 / 3}}{\Gamma\left(\frac{1}{3}\right) \Gamma\left(\frac{5}{6}\right)}(-2 i)^{n+\frac{2}{3}} \Gamma\left(n+\frac{1}{6}\right),(60)$ as $n \rightarrow \infty$. On the other hand

$$
b_{1, n}(z) \sim \frac{C_{21}}{2 \pi i} \frac{b_{2,0}(z) \Gamma(n+\nu)}{\left(f_{2}(z)-f_{1}(z)\right)^{n+\nu}}, \quad \text { as } n \rightarrow \infty .
$$

where $C_{21}$ is the Stokes multiplier relating to the switching on across Stokes line (b) (see figure 2) of the asymptotic expansion of $y_{2}(z)$ by the asymptotic expansion of function $y_{1}(z)$.

Hence, by comparison of (60) and (61), we see that $\nu=\frac{1}{6}$. In addition, since

$$
\frac{b_{2,0}(z) \Gamma\left(n+\frac{1}{6}\right)}{\left(f_{2}(z)-f_{1}(z)\right)^{n+\frac{1}{6}}} \sim \mathrm{e}^{1 / 3}(-2 i)^{n+\frac{1}{2}} \Gamma\left(n+\frac{1}{6}\right)(z-i)^{-2 n-2 / 3}, \quad \text { as } z \rightarrow i,
$$


it follows that

$$
\frac{C_{21}}{2 \pi i}=\frac{\mathrm{e}^{-\pi i / 12} 2^{1 / 6}}{\Gamma\left(\frac{1}{3}\right) \Gamma\left(\frac{5}{6}\right)} .
$$

If one first crosses Stokes line $(a)$ and then Stokes line $(b), \hat{y}_{0}(z)$ first switches on $K_{1,0} y_{1}(z)$ and then $y_{1}(z)$ switches on $C_{21} y_{2}(z)$. Hence using (42), it follows that

$$
K_{2,0}=C_{21} K_{1,0}=2 \sqrt{3} \mathrm{e}^{5 \pi i / 6}, \quad \text { and that } \mu_{2}=\mu_{1}+\nu=0 .
$$

\section{Location and activity of Stokes and higher order Stokes curves}

The purpose of this section is to discuss the Stokes phenomenon and HOS and the related resurgence properties in the late terms of the expansions of the solutions.

For the purpose of this section the $C_{j}$ can be regarded as constants. If they were to be functions of $\varepsilon>0$, this would have no effect on the discussion below.

From section 3 it seems that (22) has a two-parameter family of solutions with expansions that start

$y(z ; \varepsilon) \sim \mathrm{e}^{-f_{0}(z) / \varepsilon} \sum_{s=0}^{\infty} a_{s}(z) \varepsilon^{s}+C_{1} \mathrm{e}^{-f_{1}(z) / \varepsilon} \sum_{s=0}^{\infty} \beta_{1, s}(z) \varepsilon^{s+\frac{1}{6}}+C_{2} \mathrm{e}^{-f_{2}(z) / \varepsilon} \sum_{s=0}^{\infty} \beta_{2, s}(z) \varepsilon^{s}$,

as $\varepsilon \rightarrow 0^{+}$, where in this paper $f_{0}(z) \equiv 0$.

The coefficients $\beta_{1, s}(z)$ and $\beta_{2, s}(z)$ satisfy recurrence relations and are found in sections 4 and 5 . The presence of the power of $1 / 6$ that appears in the $\beta_{1, s}(z)$ series is explained in section 4 . Higher order terms forming a transseries are also present, but it is sufficient for the time being to examine the properties of (65).

The two exponentially prefactored terms in (65) can be switched on in the complex $z$-plane as a result of Stokes phenomena involving the $a_{s}(z)$. The resurgence properties [13] of $a_{s}(z)$ thus suggest an expansion of the form

$$
a_{n}(z) \sim \frac{1}{2 \pi i} \sum_{s=0}^{\infty} \frac{\beta_{1, s}(z) \Gamma\left(n-s-\frac{1}{6}\right)}{\left(f_{1}(z)\right)^{n-s-\frac{1}{6}}}+\frac{1}{2 \pi i} \sum_{s=0}^{\infty} \frac{\beta_{2, s}(z) \Gamma(n-s)}{\left(f_{2}(z)\right)^{n-s}},
$$

as $n \rightarrow \infty$. This is $(16)$ with $\mu_{1}=1 / 6$ and $\mu_{2}=0$.

Using this expansion, we may determine $k_{1}$ : note that since $a_{n}(z)$ has a pole at $z=-i$ of order $2 n-1$, it follows from a comparison of (29) and (66) that $k_{1}=0$ and so

$$
f_{1}(z)=-\frac{1}{2} i(z+i)^{2} \text {. }
$$

In section 5 we have shown that $f_{2}(i)=f_{1}(i)$ and hence $k_{2}=0$, thus $f_{2}(z)=2 z$.

Consider equation (65). It is well known, see for example [2], that the multipliers $C_{1}, C_{2}$, may change across an active Stokes line in the $z$ plane. A Stokes line may occur at

$$
\Im\left(f_{i}(z)-f_{j}(z)\right)=0, \quad i \neq j .
$$

If a Poincaré definition of the asymptotic expansion is used, traversal of anti-Stokes lines where $\Re\left(f_{i}(z)-f_{j}(z)\right)=0$, may also lead to an apparent change of prefactors and 


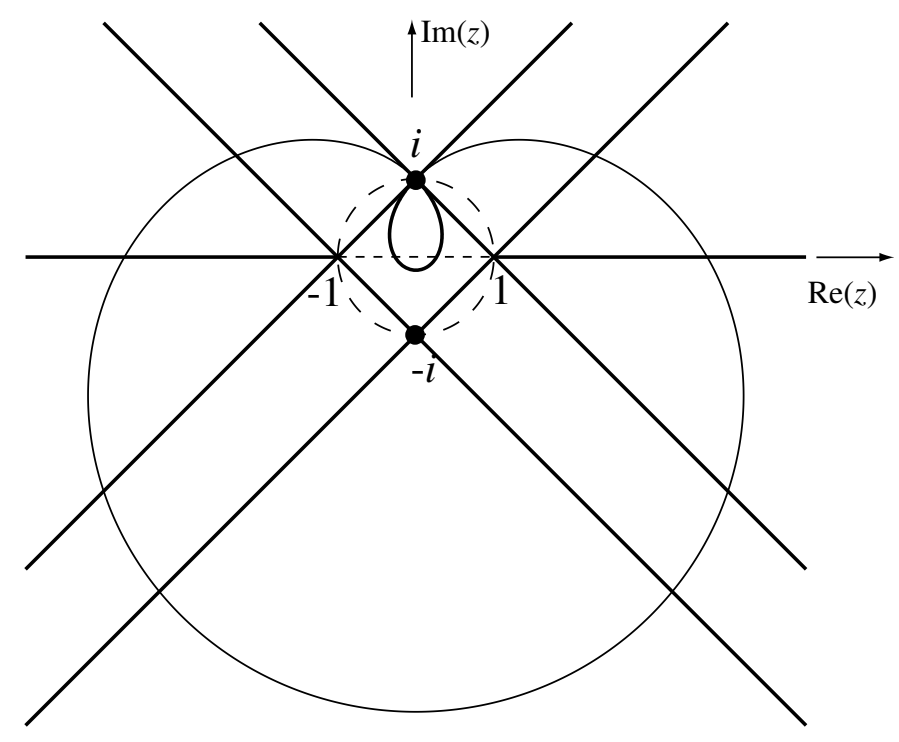

Figure 1. Active Stokes curves (the straight lines), higher order Stokes curve (the unit circle) for the asymptotic terms of (65) and the curve where $\left|f_{1}(z)\right|=\left|f_{2}(z)\right|$ (the union of the "cardioid" outside and "teardrop" inside the unit circle). The dashed horizontal line is an inactive Stokes line (see below). The black dots at $\pm i$ are turning points. The points \pm 1 are regular points where Stokes lines cross.

the apparent form of asymptotic expansions as dominance changes. However this is an artefact of the exclusion of exponentially small terms in the Poincaré approach. The exponential asymptotic approach fundamentally extends the Poincaré both in terms of accuracy and uniformity and shows that there is no such change in prefactors of an exponentially accurate expansion across an anti-Stokes line.

Then, using (68), figure 1 shows the potential Stokes curves (straight lines) across which the multipliers $C_{1}, C_{2}$, of the asymptotic terms in (65) may change according to (68).

It is now known $([4],[5],[6],[2],[1],[7])$, that in the presence of three or more asymptotic contributions with independent exponential prefactors as in (65), knowledge of the existence and location of Stokes curves emanating from turning points alone is not sufficient to understand the global connection problem and resolve all the changes of the $C_{1}$ and $C_{2}$ as the $z$ plane is traversed. Under these circumstances the activity of Stokes curves may be switched on or off at regular points in the $z$ plane, leading to apparent extra or missing changes $C_{1}$ and $C_{2}$.

One way to explain this is to introduce the concept of a higher order Stokes curve. This is the locus of points for which

$$
\left(\frac{f_{0}(z)-f_{1}(z)}{f_{1}(z)-f_{2}(z)}\right)>0
$$

It can be shown, see for example [2], that it is across such curves in the $z$-plane that the activity of a Stokes curve changes. We have termed such a change in activity 
of Stokes curve as a higher order Stokes Phenomenon.

Stokes curves usually connect turning points, or turning points and infinity. Turning points are where the exponents in the exponential prefactors degenerate and the coefficients in asymptotic series diverge.

A consequence of the change in activity of a Stokes curve across a higher order Stokes curve is that the inactive continuation of the Stokes curve may encounter a point that should otherwise be a turning point where $f_{i}(z)=f_{j}(z)$, but where the terms in (65) do not diverge. Such points are called virtual turning points ([5], [6], [7]).

Virtual turning points have been shown [7] to be associated with the presence of "smoothed shocks", where there is a rapid (monotonic) local transition between different values of the solution. All of these effects can be studied by considering the related singularity structure in the Borel plane representation of the problem. When a higher order Stokes phenomenon takes place, some of the singularities in the Borel plane move to different Riemann sheets. For details see, for example [2].

Which of Stokes curves are active and where depends on the initial data taken for the specific solution of (22) and location of that data.

For the specific $f_{j}(z)$ studied, the higher order Stokes curve (69) corresponds to the circle $|z|=1$.

The relative dominance of the two divergent series in the right hand side of (66) changes as the curve $\left.\left|f_{1}(z)\right|=\mid f_{2}(z)\right) \mid$ is crossed. This curve is the union of the "cardioid" and "teardrop" shapes in figure 1(outside and inside, respectively the unit circle). Inside the teardrop, $\left|f_{1}(z)\right|>\left|f_{2}(z)\right|$. Outside the cardioid $\left|f_{1}(z)\right|>\left|f_{2}(z)\right|$. In the remaining region we have $\left|f_{1}(z)\right|<\left|f_{2}(z)\right|$.

Inside the higher order Stokes curve the series in $\beta_{2, s}(z)$ cannot be present in (66). Otherwise, since $f_{2}(0)=0, a_{n}(z)$ would blow up at the origin, which they do not. In section 5 we have shown that this series is present in (66) outside the higher order Stokes curve, where $|z|>1$.

Hence outside the cardioid region the $\beta_{2, s}(z)$ series dominates the $\beta_{1, s}(z)$ series in (66). Conversely inside the cardioid the series in $\beta_{1, s}(z)$ dominates the right hand side of (66). (Since the $\beta_{2, s}(z)$ is absent inside the higher order Stokes curve, in this case the teardrop portion of the curve is irrelevant.)

Note that there are turning points at both $-i$ and $+i$. The former occurs because $f_{1}(-i)=f_{0}(-i)=0$. The latter occurs because $f_{2}(i)=f_{1}(i)$. Note that in the region $\{z \in \mathbb{C}: \Re z>0$ and $|\Im z|<1\}$, the asymptotic terms prefactored by $\mathrm{e}^{-f_{0}(z) / \varepsilon}$ dominate those prefactored by $\mathrm{e}^{-f_{1}(z) / \varepsilon}$, which in turn dominate those prefactored by $\mathrm{e}^{-f_{2}(z) / \varepsilon}$. The latter terms are thus not just subdominant, but sub-subdominant. If these terms were to be neglected, then not only the presence of the turning point at $z=+i$ would be overlooked, but the Stokes line emanating from it would also evaporate. An immediate consequence of this would be that in passing from $z>1$ to $z<1$, no contribution from series prefactored by $\mathrm{e}^{-f_{2}(z) / \varepsilon}$ could be switched on. This would result in a totally incorrect approximation to the solution for values of $z<0$, where the $\mathrm{e}^{-f_{2}(z) \varepsilon}$ terms dominate the asymptotics. 
Finally, because of the presence of the higher order Stokes curve, (which also switches off the activity of the Stokes line between -1 and +1 along the real axis), the point $z=0$, where $f_{2}(0)=f_{0}(0)=0$, is a so-called "virtual turning point".

\section{Exponential sensitivity to initial data: locking-on to the chosen solution}

Before we further explore the asymptotic details of the solutions, we must first address a significant numerical issue, generically encountered in nonlinear odes: the exponential sensitivity to initial data.

Clearly it is possible to substitute chosen initial data into a solver routine to generate numerical solutions of (22), up to the accuracy of the algorithm.

However our goal is to compare the exponentially asymptotic approximations with specific numerical solutions, first to demonstrate the validity of the asymptotics, secondly to confirm the asymptotic predictions of the activity of Stokes lines and the presence of a higher order Stokes phenomenon and thirdly (and consequently) to provide a general analytical asymptotic explanation of the underlying solutions. We therefore have to make sure that the initial data and algorithms for the numerical routines are consistent and calibrated with the analytical asymptotic approximations.

We seek to track the numerical solution that has the formal expansion (3) with $a_{0}(z)=\alpha_{-}(z)$ and with $C_{1}=C_{2}=0$ in (65). This is a solution for which there are no exponentially small contributions prefactored by $\mathrm{e}^{-f_{j}(z) / \varepsilon}, j=1,2$ present at the initial data point. We thus have to find the correct initial data that will (up to the accuracy of the numerical solver) generate this specific solution. This is a subtle point that is easy to overlook.

For a wide class of asymptotic expansions, it is known that if they are "optimally" truncated at the term that is smallest in magnitude, they can generate exponential accurate approximations [3]. Guided by this, when seeking to track a specific numerical solution to a (nonlinear) equation that is exponentially sensitive to initial data, a first thought might be to seed a numerical solver routine with initial conditions based on an optimal truncation of the asymptotic expansion we wish to follow.

However for (22) this proves not to be sufficient. We have to move to a higher level of precision in the specification of the initial data that is sufficient to resolve the presence or otherwise of any sub-subdominant exponential contribution. Fortunately, hyperasymptotics $([9],[10],[11],[13],[14],[15])$ provides the solution. For details on the optimal number of terms and hyperasymptotics for nonlinear ordinary differential equations see [14] and [15]. The general formula for the optimal number of terms and other hyperasymptotic formulae we use here are summarised in appendix A.

First we demonstrate the inadequacies of the conventional asymptotic and optimal truncation approaches.

Optimally truncated asymptotic expansions have exponentially small error estimates (see, e.g., [13], [14] and [15]). The optimal number of terms in the asymptotic expansion $\hat{y}_{0}(z)$ depends on the active small exponentials, i.e., those that can have their 
coefficient changed in a sector containing $z$ via a Stokes phenomenon (but in this case are not yet contributing at the initial data point $z_{0}$ where $C_{1}$ and $C_{2}$ are currently both $0)$. The exponentials are $\exp \left(-f_{j}(z) / \varepsilon\right), j=1,2$, and hence, the optimal number of terms in $\hat{y}_{0}(z)$ is approximately $\min \left(\left|f_{1}(z) / \varepsilon\right|,\left|f_{2}(z) / \varepsilon\right|\right)$, at a point $z$. However, for $|z|<1$ the $\beta_{2, s}(z)$ cannot be present in (66) (due to the lack of divergence of $a_{n}(0)$ ) and for $|z|<1$ the optimal number of terms in $\hat{y}_{0}(z)$ is approximately $\left|f_{1}(z) / \varepsilon\right|$. Hence, for $|z|<1$ the optimally truncated expansion for the chosen $y(z ; \varepsilon)$ is

$$
y(z ; \varepsilon)=\sum_{s=0}^{N-1} a_{s}(z) \varepsilon^{s}+\mathcal{O}\left(\mathrm{e}^{-N}\right), \quad \text { as } \varepsilon \rightarrow 0^{+}, \quad N=\left\lfloor\left|f_{1}(z) / \varepsilon\right|\right\rfloor,
$$

where $\lfloor|\cdots|\rfloor$ denotes the integer part, see (A.2).

First, we show that a naïve asymptotic approach is not sufficient.

If we take $\varepsilon=\frac{1}{10}$, start at the point $z_{0}=0.8$ and take only 4 terms in the asymptotic approximation (which is not an optimal truncation) we have

$$
\begin{aligned}
& y\left(z_{0} ; \frac{1}{10}\right) \approx \sum_{s=0}^{3} a_{s}\left(z_{0}\right) \varepsilon^{s} \approx-0.98325330+0.22606915 i \\
& y^{\prime}\left(z_{0} ; \frac{1}{10}\right) \approx 0.01007179+0.35987558 i
\end{aligned}
$$

We use a Taylor series method (see $\S 3.7($ ii) in [23]) to integrate the differential equation, here using 500 steps and 10 Taylor coefficients to walk from $z_{0}=0.8$ to $z_{1}=0.3$. (The accuracy of this will be confirmed below.) The result is

$$
y\left(z_{1} ; \frac{1}{10}\right) \approx 1.91634981-0.21149378 i .
$$

As no Stokes lines have been crossed in the interval $\left[z_{1}, z_{0}\right]$ (see figure 1), the solution that we are looking for should still have the same form of asymptotic expansion (71). Hence, the 'exact' solution should be approximated asymptotically by

$$
y\left(z_{1} ; \frac{1}{10}\right) \approx \sum_{s=0}^{4} a_{s}\left(z_{1}\right) \varepsilon^{s} \approx-0.99703133+0.05045106 i
$$

(where we have taken 5 terms here as $N=\left\lfloor\left|f_{1}\left(z_{1}\right) / \varepsilon\right|\right\rfloor=5$ ).

Clearly the numerical results (73) and (74) are very different. Either something has gone wrong during the numerical integration and/or a naïve Poincaré approximation is insufficient.

Thence, we turn to initial data specified at $z_{0}=0.8$ by an asymptotic expansion truncated at the optimal number of terms, $N=\left\lfloor\left|f_{1}\left(z_{0}\right) / \varepsilon\right|\right\rfloor=8$. We have

$$
\begin{aligned}
& y\left(z_{0}, \frac{1}{10}\right) \approx \sum_{s=0}^{7} a_{s}\left(z_{0}\right) \varepsilon^{s} \approx-0.98354251+0.22566150 i \\
& y^{\prime}\left(z_{0}, \frac{1}{10}\right) \approx 0.01326763+0.36006092 i
\end{aligned}
$$

The same numerical integration procedure then generates

$$
y\left(z_{1}, \frac{1}{10}\right) \approx-1.46444502+1.53563049 i .
$$


Hence, there is hardly an improvement, even with optimally truncated, exponentially accurate approximations for the initial data.

An obvious cause for this lack of agreement could be that the numerical integration algorithm is not sufficiently accurate. However we can confirm that this is not (systematically) the case and resolve the contradiction by going beyond optimal truncation and employing a hyperasymptotic approach. Such an approach can generate expansions of better-than-exponential accuracy, that can be used to unambiguously resolve the presence, or not, of exponentially small contamination in the exponential data arising from the terms prefactored by $C_{1}$ and $C_{2}$ in (35).

For details on hyperasymptotic expansions and the definition of the hyperterminant function $F^{(1)}$, see appendix A.

The level 1 hyperasymptotic expansion for $y(z ; \varepsilon)$ with $C_{1}=C_{2}=0$ is

$y(z ; \varepsilon)=\sum_{s=0}^{N-1} a_{s}\left(z_{0}\right) \varepsilon^{s}+\frac{\varepsilon^{N-1}}{2 \pi i} \sum_{m=0}^{M-1} \beta_{1, s}(z) F^{(1)}\left(\frac{1}{\varepsilon} ; \begin{array}{c}N-s-\frac{1}{6} \\ -f_{1}(z)\end{array}\right)+\mathcal{O}\left(\mathrm{e}^{-N}\right)$, as $\varepsilon \rightarrow 0^{+},($

where $M=\left\lfloor\min \left(\left|f_{1}(z) / \varepsilon\right|,\left|\left(f_{1}(z)-f_{2}(z)\right) / \varepsilon\right|\right)\right\rfloor, N=\left\lfloor\left|f_{1}(z) / \varepsilon\right|\right\rfloor+M$.

If we start with the level 1 hyperasymptotic approximation to the initial data at $z_{0}=0.8$ with $\varepsilon=\frac{1}{10}$, we thus have

$$
\begin{aligned}
y\left(z_{0}, \frac{1}{10}\right) & \approx \sum_{s=0}^{15} a_{s}\left(z_{0}\right) \varepsilon^{s}+\mathrm{e}^{\pi i / 3} \frac{\varepsilon^{15}}{2 \pi i} \sum_{s=0}^{7} \beta_{1, s}\left(z_{0}\right) F^{(1)}\left(\frac{1}{\varepsilon} ; \begin{array}{c}
16-s-\frac{1}{6} \\
-f_{1}\left(z_{0}\right)
\end{array}\right) \\
& \approx-0.98359559+0.22575467 i \\
y^{\prime}\left(z_{0}, \frac{1}{10}\right) & \approx 0.01263472+0.35905425 i .
\end{aligned}
$$

Note the $\mathrm{e}^{\pi i / 3}$ in front of the $F^{(1)}$ sum. This is a consequence of the multi-valuedness of the $\sigma$ power in $(A .9)$.

Using these initial values in the numerical integration yields

$$
y\left(z_{1}, \frac{1}{10}\right) \approx-0.99658777+0.05046433 i .
$$

A comparison of (74) with (80) confirms that, at least on the interval $z_{1}<z<z_{0}$ the hyperasymptotic precision in the initial data allows us to accurately compare the numerical and analytical solution. By implication this also suggests that the numerical algorithm is relatively accurate and stable.

If we wished to continue the numerical tracking over larger regions of the $z$-plane, further hyperasymptotic refinement of the initial data might be required. The procedure for higher level hyperasymptotic approximation of functions at $z_{0}$ can be found in [13].

It might be a bit surprising that optimal truncation is not enough. To understand why we consider further the initial data (75). This is a perfectly valid set of initial data, but not for the solution we wish to track asymptotically. Just what is the solution corresponding to $(75)$ ?

We compare the results obtained from the initial data (75) with the better-thanexponentially accurate hyperasymptotic representation (79) at the initial point $z_{0}$ via 


$$
\begin{aligned}
-0.98354251+0.22566150 i= & -0.98359559+0.22575467 i \\
& +C_{1} \mathrm{e}^{-f_{1}\left(z_{0}\right) / \varepsilon} \sum_{s=0}^{7} \beta_{1, s}\left(z_{0}\right) \varepsilon^{s}+C_{2} \mathrm{e}^{-f_{2}\left(z_{0}\right) / \varepsilon} \beta_{2,0}\left(z_{0}\right), \\
0.01326763+\quad 0.36006092 i= & 0.01263472+0.35905425 i \\
+ & \frac{d}{d z}\left(C_{1} \mathrm{e}^{-f_{1}\left(z_{0}\right) / \varepsilon} \sum_{s=0}^{7} \beta_{1, s}\left(z_{0}\right) \varepsilon^{s}+C_{2} \mathrm{e}^{-f_{2}\left(z_{0}\right) / \varepsilon} \beta_{2,0}\left(z_{0}\right)\right) .
\end{aligned}
$$

Note that on the right-hand side of (81) the first numerical term was obtained in (79), and the second series on the right-hand side of (81) is an optimally truncated series. The accuracy of these expansions makes the final sub-subdominant term numerically just visible. If we were to consider more terms in the $C_{2}$ part of $(81)$, we would have to go to even higher precision and use a level 2 hyperasymptotic expansion [9], [13].

We can solve this system of equations and obtain

$$
C_{1}=0.24612117+0.09937446 i, \quad C_{2}=122.25743+10.11636 i .
$$

Using these values in the resummed transseries approximation (35) we have

$$
\begin{aligned}
a_{0}\left(z_{1}\right)+ & \frac{C_{1} \beta_{1,0}\left(z_{1}\right) \mathrm{e}^{-f_{1}\left(z_{1}\right) / \varepsilon}+C_{2} \beta_{2,0}\left(z_{1}\right) \mathrm{e}^{-f_{2}\left(z_{1}\right) / \varepsilon}}{1+C_{1} \beta_{1,0}\left(z_{1}\right) \mathrm{e}^{-f_{1}\left(z_{1}\right) / \varepsilon} / f_{1}^{\prime}\left(z_{1}\right)+C_{2} \beta_{2,0}\left(z_{1}\right) \mathrm{e}^{-f_{2}\left(z_{1}\right) / \varepsilon} / f_{2}^{\prime}\left(z_{1}\right)} \\
& =-1.4779361+1.5940551 i
\end{aligned}
$$

This result is much closer at $z_{1}$ to the numerically integrated result (77). Hence we conclude that the initial data (75) is consistent with an asymptotic approximation with the approximate values for $C_{1}$ and $C_{2},(83)$.

The magnitude of $C_{2}$ shows that by the end of the numerical integration at $z_{1}$, although we are still in a region where the exponentials are small, the combination $C_{2} \mathrm{e}^{-f_{2}\left(z_{1}\right) / \varepsilon}$ can be numerically significant.

This illustrates a further significant point. Although z-dependent exponential contributions in transseries (or their resummations) may be asymptotically subdominant, the $z$-independent constants $C_{1}$ and $C_{2}$ associated with them may be sufficiently large in magnitude that they are, nevertheless, numerically significant.

Exponential sensitivity of (nonlinear) problems to initial data is a well-known problem. However the results of this section demonstrate how a hyperasymptotic approach might, in principle, be used to track specific solutions over a finite interval.

\section{Numerical verification of activity of Stokes lines}

We now verify numerically the form of the asymptotics and the numerical values of the Stokes constants, together with the activity of Stokes lines $(a)$ and $(b)$ in figure 2. It is possible to check the activity of the other Stokes lines in figure 2 in a similar way to what follows, using alternative comparison points. 

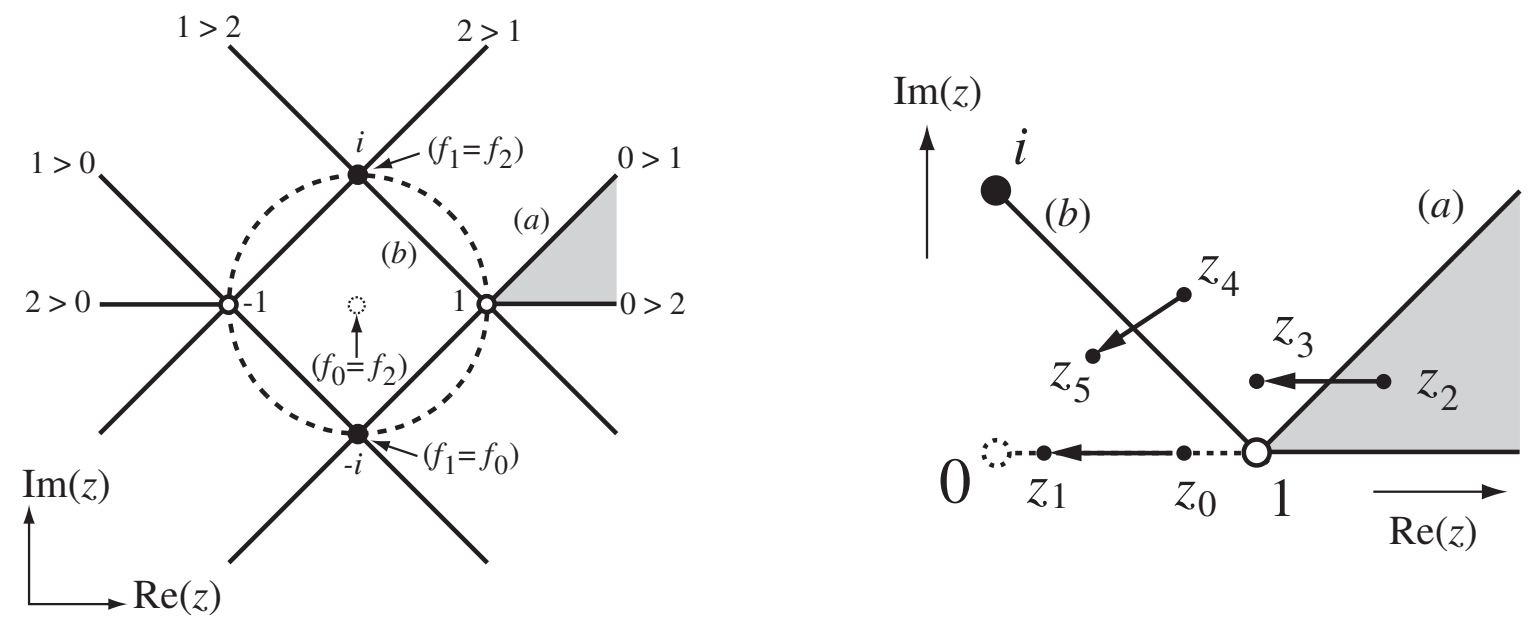

Figure 2. Left hand plot: active Stokes curves, the higher-order Stokes curve (dashed), the turning points (black dots), Stokes crossing points (white dots) and the virtual turning point (dashed central dot). The numbers $j>k$ by each Stokes line denotes the relative dominance of the series prefactored by $f_{j}(z)$ over $f_{k}(z)$. A series prefactored by an exponential $f_{j}(z)$ forces a change in the Stokes constant $C_{k}$ as the $j>k$ Stokes curve is traversed. Right hand plot: zoomed version of northeast corner of left-hand plot to give a summary of the relative locations of the points $z_{i}, i=0, \cdots, 5$, used in the numerical calculations and verification of the activity of the Stokes lines.

\subsection{Numerical verification on crossing Stokes line (a)}

Our starting sector is $\{z \in \mathbb{C}: \Re z>1$ and $0<\Im z<\Re z-1\}$, the shaded sector in figure 2 . In this sector we take the solution of $(22)$ with asymptotic expansion $\hat{y}_{0}(z ; \varepsilon)$. Hence, this solution should have no active exponentially small terms. As shown in section 7 , numerically we can achieve this by using the level 1 hyperasymptotic expansion for this solution.

In the following hyperasymptotic calculations, we shall truncate the series at the optimal number of terms, as determined by the method outlined in appendix A.

Starting at $z_{2}=1.2+0.1 i$ with $\varepsilon=\frac{1}{6}$ we have

$$
\begin{aligned}
y\left(z_{2} ; \frac{1}{6}\right) & \approx \sum_{s=0}^{13} \frac{a_{s}\left(z_{2}\right)}{6^{s}}+\frac{\varepsilon^{13}}{2 \pi i} \sum_{s=0}^{5} \beta_{1, s}\left(z_{2}\right) F^{(1)}\left(\frac{1}{\varepsilon} ; \begin{array}{c}
14-s-\frac{1}{6} \\
-f_{1}\left(z_{3}\right)
\end{array}\right) \\
& \approx-1.00509821+0.34416732 i \\
y^{\prime}\left(z_{2} ; \frac{1}{6}\right) & \approx-0.00243012+0.36793122 i .
\end{aligned}
$$

Again we use a Taylor series method to integrate the differential equation, here using 500 steps and 10 Taylor coefficients to walk from $z_{2}=1.2+0.1 i$ to $z_{3}=1.0+0.1 i$ across the Stokes line $(a)$. We obtain with $\varepsilon=\frac{1}{6}$,

$$
\begin{aligned}
\sum_{s=0}^{11} a_{s}\left(z_{3}\right) \varepsilon^{s}+\mathrm{e}^{\pi i / 3} \frac{\varepsilon^{11}}{2 \pi i} \sum_{s=0}^{4} \beta_{1, s}\left(z_{3}\right) F^{(1)}\left(\frac{1}{\varepsilon} ; \begin{array}{c}
12-s-\frac{1}{6} \\
-f_{1}\left(z_{3}\right)
\end{array}\right) & \approx-1.00731156+0.26995479 i \\
\operatorname{ditto}+\mathrm{e}^{-f_{1}\left(z_{3}\right) / \varepsilon} \sum_{s=0}^{4} \beta_{1, s}\left(z_{3}\right) \varepsilon^{s+\frac{1}{6}} & \approx-1.00561125+0.26905061 i,
\end{aligned}
$$




$$
\left.y\left(z_{3} ; \frac{1}{6}\right)\right|_{\text {num }} \quad \approx-1.00561380+0.26905034 i,
$$

where the subscript "num" denotes the result of the numerical integration. The numerics clearly shows that a contribution of the form $K^{-f_{1}(z) / \varepsilon} y_{1}(z ; \varepsilon)$ is switched on via a Stokes phenomenon across $(a)$ with $K=1$ and that we have found the correct values for the coefficients $\beta_{1, s}(z)$, compare (17).

Comparing (85) with (86), we observe the presence of an extra factor $\mathrm{e}^{\pi i / 3}$ in front of the series containing the $F^{(1)}$ hyperterminants. This is again a consequence of the multi-valuedness of the $\sigma$ power in $(A .9)$.

\subsection{Numerical verification on crossing Stokes line (b)}

We will now show numerically that when we cross Stokes line $(b)$ in figure 2, the function $\mathrm{e}^{-f_{1}(z) / \varepsilon} y_{1}(z ; \varepsilon)$ switches on $\mathrm{e}^{-f_{2}(z) / \varepsilon} y_{2}(z ; \varepsilon)$. In the following hyperasymptotic calculations, we shall truncate the series at the optimal number of terms, as determined by the method outlined in appendix A.

Starting at $z_{4}=0.8+0.3 i$ with $\varepsilon=\frac{1}{8}$ we have

$$
\begin{aligned}
& y_{1}\left(z_{4} ; \frac{1}{8}\right) \approx \sum_{s=0}^{7} \beta_{1, s}\left(z_{4}\right) \varepsilon^{s+\frac{1}{6}} \\
& +\mathrm{e}^{-\pi i / 3} \frac{\varepsilon^{43 / 6}}{2 \pi i} \sum_{s=0}^{3} \beta_{2, s}\left(z_{4}\right) F^{(1)}\left(\begin{array}{cc}
\frac{1}{\varepsilon} ; & 8-s+\frac{1}{6} \\
f_{1}\left(z_{4}\right)-f_{2}\left(z_{4}\right)
\end{array}\right) \\
& \approx 1.67355027+0.46012176 i, \\
& y_{1}^{\prime}\left(z_{4} ; \frac{1}{8}\right) \approx-0.30903605-1.93512600 i .
\end{aligned}
$$

We use a Taylor series method to integrate the differential equation, here using 500 steps and 10 Taylor coefficients to walk from $z_{4}$ across Stokes line $(b)$ to $z_{5}=0.7+0.2 i$ with $\varepsilon=\frac{1}{8}$ and obtain the hyperasymptotic representation of $y_{1}\left(z_{5} ; \frac{1}{8}\right)$ as

$$
\begin{aligned}
& \sum_{s=0}^{7} \beta_{1, s}\left(z_{5}\right) \varepsilon^{s+\frac{1}{6}}+\frac{\varepsilon^{43 / 6}}{2 \pi i} \sum_{s=0}^{3} \beta_{2, s}\left(z_{5}\right) F^{(1)}\left(\frac{1}{\varepsilon} ; \begin{array}{c}
8-s+\frac{1}{6} \\
f_{1}\left(z_{5}\right)-f_{2}\left(z_{5}\right)
\end{array}\right) \approx 1.43633295+0.69388479 i, \\
& \text { ditto }-\mathrm{e}^{\left(f_{1}\left(z_{5}\right)-f_{2}\left(z_{5}\right)\right) / \varepsilon} \sum_{s=0}^{3} \beta_{2, s}\left(z_{5}\right) \varepsilon^{s} \\
& \left.y_{1}\left(z_{5} ; \frac{1}{8}\right)\right|_{\text {num }} \\
& \approx 1.47430720+0.67235777 i, \\
& \approx 1.47416629+0.67231078 i \text {. }
\end{aligned}
$$

Note the minus sign in front of $\mathrm{e}^{\left(f_{1}\left(z_{5}\right)-f_{2}\left(z_{5}\right)\right) / \varepsilon}$. This is because we cross the Stokes line in the opposite direction compared with Stokes line $(a)$. The numerics clearly shows that $\mathrm{e}^{-f_{1}(z) / \varepsilon} y_{1}(z ; \varepsilon)$ is switched on via a Stokes phenomenon across $(a)$ and that we have indeed found the correct values for the Stokes multipliers. If the results of the hyperasymptotic and numerical integration had not agreed so well, it would be clear that (modulo numerical errors) the form of the asymptotic expansion including the 
Stokes multipliers and activity of the Stokes line would have been incorrect. That the hyperasymptotic and numerical results also agree suggests that the numerical integration was also stable.

\section{Uniform result: double transseries resummation}

Solutions of (22) may possess rapid transitions between different attracting behaviours, or "smoothed shocks". Their existence and location depend on the values of $C_{1}$ and $C_{2}$. If $C_{1}$ and $C_{2}$ are of $O(1)$, then the shock occurs near to $z=0$.

To demonstrate this we use the double resummation

$y(z ; \varepsilon)=\alpha_{-}(z)+\frac{C_{1} \beta_{0}(z) \mathrm{e}^{-f_{1}(z) / \varepsilon} \varepsilon^{1 / 6}+C_{2} \beta_{1,0}(z) \mathrm{e}^{-f_{2}(z) / \varepsilon}}{1+C_{1} \beta_{0}(z) \mathrm{e}^{-f_{1}(z) / \varepsilon} \varepsilon^{1 / 6} / f_{1}^{\prime}(z)+C_{2} \beta_{1,0}(z) \mathrm{e}^{-f_{2}(z) / \varepsilon} / f_{2}^{\prime}(z)}+O(\varepsilon)$,

with $C_{1}=1 C_{2}=-1$.

First we start on the right hand side of a "shock" at $z=\frac{1}{4}$ with $\varepsilon=\frac{1}{30}$. In the numerics it suffices to take $K_{j, k}=0$ for $j=1,2$ and $k>7$. Then we have

$$
\begin{aligned}
y\left(\frac{1}{4} ; \frac{1}{30}\right) \approx & \sum_{s=0}^{30} a_{s}(z) \varepsilon^{s}+\mathrm{e}^{\pi i / 3} \frac{\varepsilon^{30}}{2 \pi i} \sum_{s=0}^{14} \beta_{1, s}(z) F^{(1)}\left(\frac{1}{\varepsilon} ; \begin{array}{c}
31-s-\frac{1}{6} \\
-f_{1}(z)
\end{array}\right) \\
& +\mathrm{e}^{-f_{1}(z) / \varepsilon} \sum_{s=0}^{14} \beta_{1, s}(z) \varepsilon^{s+\frac{1}{6}}-\mathrm{e}^{-f_{2}(z) / \varepsilon} \sum_{s=0}^{14} \beta_{2, s}(z) \varepsilon^{s} \\
\approx & -0.9974759948+0.0667160847 i, \\
y^{\prime}\left(\frac{1}{4} ; \frac{1}{30}\right) \approx \quad & -0.0024353982+0.3479545146 i .
\end{aligned}
$$

We walk (500 steps, 10 Taylor coefficients) to $z=-\frac{1}{4}$, crossing the shock. In figure 3 we compare the numerical integration with the graph of (89).

$$
\sum_{s=0}^{8} a_{s}(z) \varepsilon^{s}+\frac{\beta_{0}(z) \mathrm{e}^{-f_{1}(z) / \varepsilon} \varepsilon^{1 / 6}-\beta_{1,0}(z) \mathrm{e}^{-f_{2}(z) / \varepsilon}}{1+\beta_{0}(z) \mathrm{e}^{-f_{1}(z) / \varepsilon} \varepsilon^{1 / 6} / f_{1}^{\prime}(z)-\frac{1}{2} \beta_{1,0}(z) \mathrm{e}^{-f_{2}(z) / \varepsilon}} .
$$

It is clear from figure 3 that (35) holds uniformly near the shock.

We now make remarks on several further significant issues.

First, the presence of the shock at $z=0$ is due to the growth of the contributions from $f_{2}(z)$. Starting at from a position upstream of the shock when $z>1$, these are not even the subdominant asymptotic contributions, but the sub-subdominant solutions! The observable growth of the shock is in the region where the $f_{2}(z)$ contributions grow to dominate the $a_{n}(z)$ terms, i.e., near $f_{2}(z)=f_{0}(z)=0$.

Second, it may be tempting to ignore the presence of contributions from $f_{1}(z)$ in (35). If $\varepsilon>0$, when $z>0$ both contributions from $f_{1}(z)$ and $f_{2}(z)$ are exponentially small. For $z<0$ the $f_{2}(z)$ terms dominate the $f_{1}(z)$ terms. However near $z=0$ when $f_{1}(0)=i / 2$ (the imaginary axis is an anti-Stokes line for $f_{1}$ ) its oscillatory contribution in (35) is briefly $\mathcal{O}(1)$, as can be seen in the imaginary part of figure 3.

Third, there is no singularity in (35) at the shock position, where $f_{2}(0)=f_{0}(0)$ arising from the dependence of the late $a_{n}(z)$ on the $c_{s}(z)(c f .(66))$. As in [7], the 


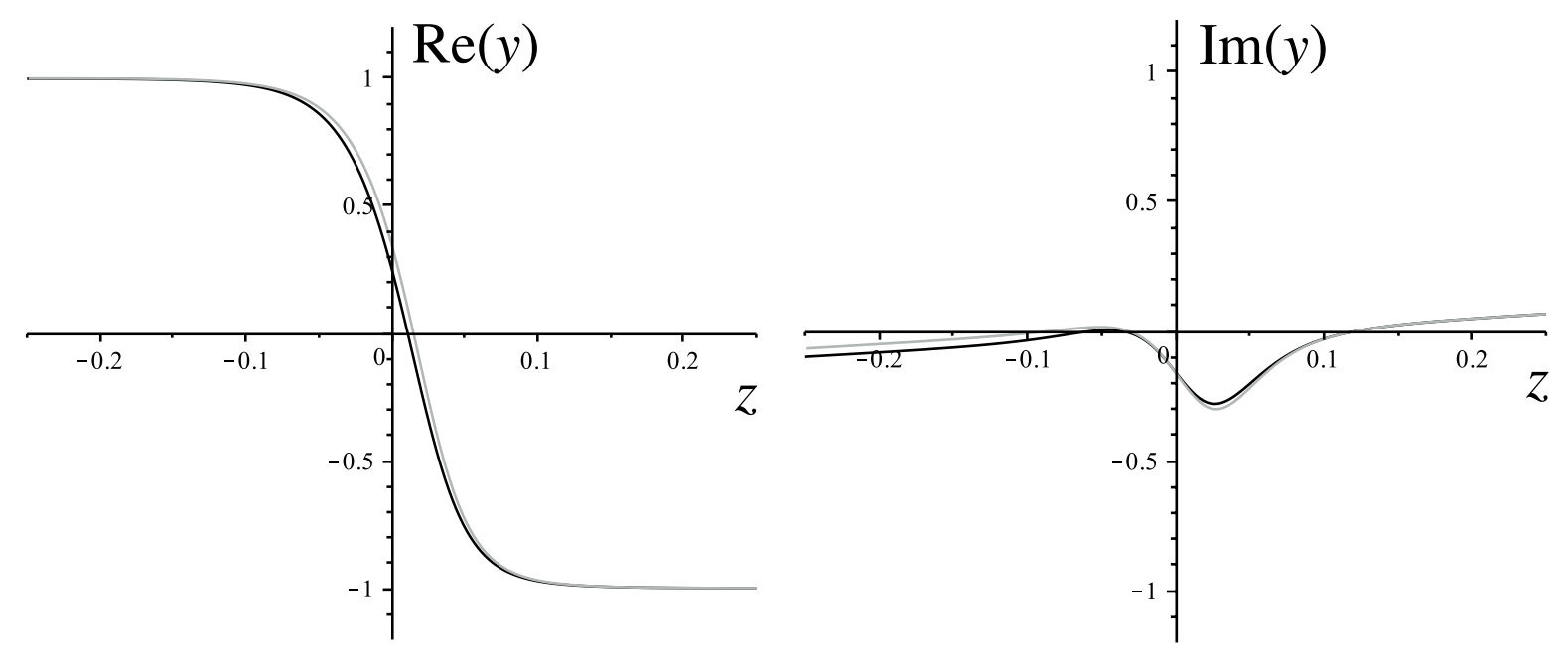

Figure 3. Numerical (grey) and asymptotic curves: real part left and imaginary part right, for $\varepsilon=1 / 30$.

presence of the higher order Stokes curve $|z|=1$ has forced the singularity $f_{2}$ to move onto a different Riemann sheet from the point $f_{0}$. Therefore there is no coalescence of these singularities even though $f_{2}(0)=f_{0}(0)$. The point $z=0$ is a virtual turning point.

The double resummation can also be used to approximate the location of poles of solutions. Taking again the case $C_{1}=1$ and $C_{2}=-1$, according to (91) we should expect a pole near

$$
1+\beta_{0}(z) \mathrm{e}^{-f_{1}(z) / \varepsilon} \varepsilon^{1 / 6} / f_{1}^{\prime}(z)-\beta_{1,0}(z) \mathrm{e}^{-f_{2}(z) / \varepsilon} / f_{2}^{\prime}(z)=0 .
$$

When, for example, $\varepsilon=1 / 15$ this form suggests that there should be a pole near $z=0.01446849+0.11425470 i$. We can easily verify this by numerical integration around a loop containing this location, as follows.

We start again at the point $z=\frac{1}{4}$ and again compute the initial values at this point via the level 1 hyperasymptotic approximation. Via Taylor series method we integrate the differential equation and store the values of $y(z ; \varepsilon)$ and $y^{\prime}(z ; \varepsilon)$ for a (large) number of points along the sides of the rectangle with vertices $0.1,-0.1,-0.1+0.14 i$ and $0.1+0.14 i$. We use these values in the extended Simpson's rule to integrate $y^{\prime}(z ; \varepsilon) /(2 \pi i y(z ; \varepsilon))$ around this rectangle in the order mentioned above. The result is 1 with a precision of more than 10 digits. Hence, there is one pole in this square.

Finally, we integrate $z y^{\prime}(z ; \varepsilon) /(2 \pi i y(z ; \varepsilon))$ along this curve and obtain for the pole position $z=0.02568526+0.11888311 i$. Thus our previous approximation is within an error $\mathcal{O}(\varepsilon)$.

Figure 4 demonstrates the ability of (89) to interpolate between all possible solutions for given starting values of $C_{1}$ and $C_{2}$ in the shaded region of figure 2. Starting with a dominant series $y(z ; \varepsilon) \sim \alpha_{-}(z)$ in the shaded region, it is possible to observe which combinations of $C_{1}$ and $C_{2}$ lead to homoclinic $\left(y(z ; \varepsilon) \sim \alpha_{-}(z)\right)$ or heteroclinic $\left(y(z ; \varepsilon) \sim \alpha_{0}(z)\right.$ or $\left.\alpha_{+}(z)\right)$ solutions as $z \rightarrow-\infty$. 


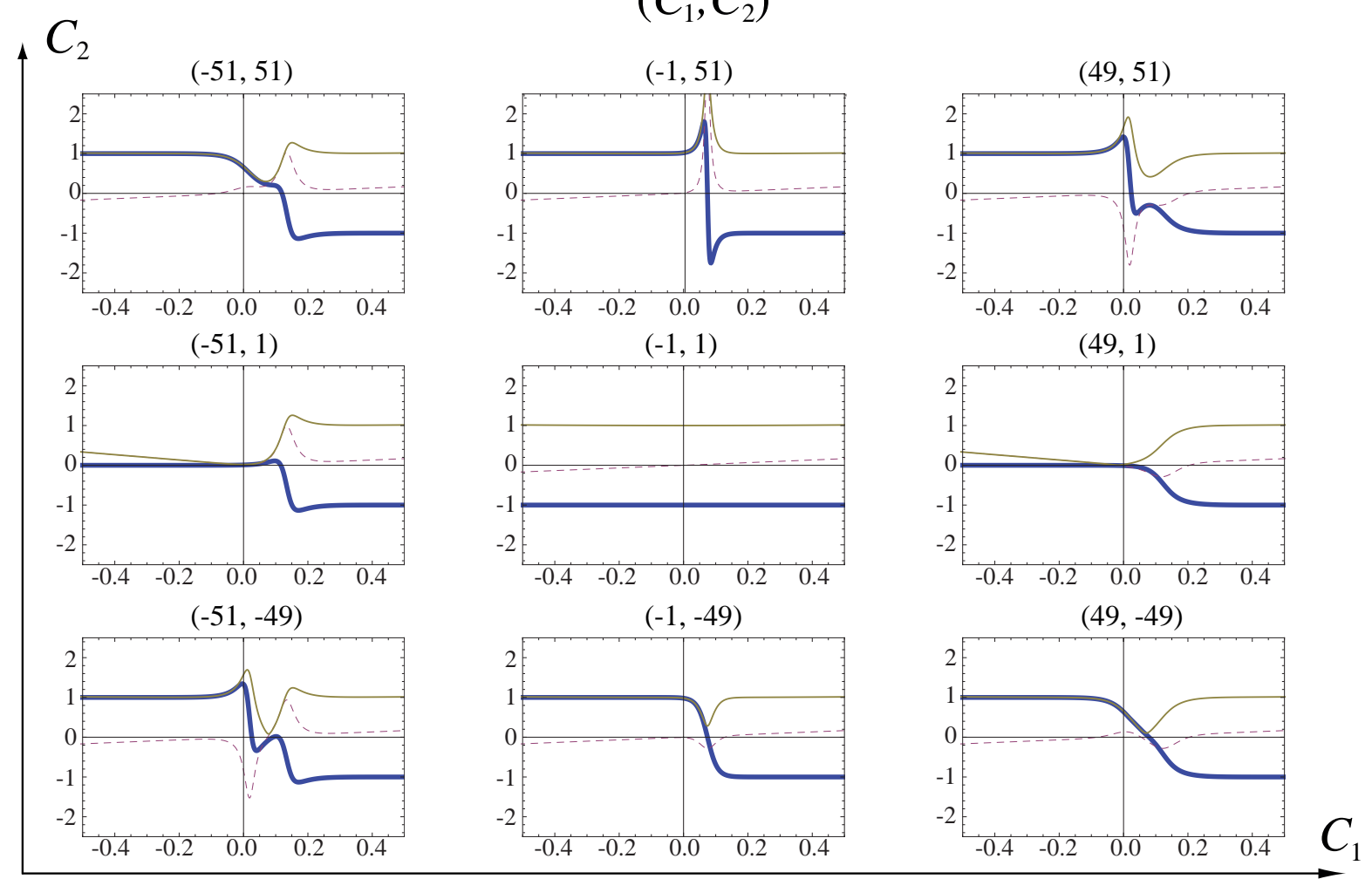

Figure 4. Plots of the asymptotic representations (89) for $\varepsilon=1 / 30$ for different starting values of $C_{1}$ and $C_{2}$ in the shaded region of figure 2. The real part is in bold, the imaginary part is dotted, the absolute value is the thin solid line. To save space, only the range of $z$ where significant change in the real part of the solution is shown.

Note that the values of $C_{1}$ and $C_{2}$ in (89) change between $z>1$ and $z<1$. For example the starting values $C_{1}=-1, C_{2}=+1$ in the shaded region, change on crossing the Stokes lines from $\pm i$ to take the values $C_{1}=0$ and $C_{2}=0$ in the region $z<1$. This generates the solution $y(z ; \varepsilon) \sim \alpha_{-}(z)$ for $z \rightarrow \pm \infty$.

More generally, it is important to realise that the middle row of plots in figure 4 could not stably be obtained by direct forward or backwards numerical integration due to the presence of growing exponentials, as revealed by the asymptotics in section 3 . The result is that because of the presence of a stable attracting solution with asymptotic values of \pm 1 as $z \rightarrow \mp \infty$, finite precision numerical techniques cannot easily be made to follow the chosen solutions for large values of $z$. All these plots correspond to solutions that start with $C_{2}=+1$ in the shaded region of figure 2. As the Stokes lines from $\pm i$ are crossed, $C_{2}$ changes to 0 : terms in $\mathrm{e}^{-f_{2}(z) / \varepsilon}$ are therefore absent in all these solutions for $z \rightarrow-\infty$ and so cannot dominate the behaviour of the solution there. Even if hyperasymptotically-seeded initial data were to be used, the numerical solver would, over a sufficiently extended range as $z \rightarrow-\infty$, be attracted to lock on numerically to follow $\alpha_{+}(z),(\Re(z) \rightarrow+1)$, rather than the correct $y(z ; \varepsilon) \sim \alpha_{-}(z),(\Re(z) \rightarrow-1)$ or $\alpha_{0}(z),(\Re(z) \rightarrow 0)$. That we can obtain the true behaviour for these $C_{1}$-parameter 
family of solutions is due to the resummation (89). Note that other one-parameter families solutions also exist.

The values of $C_{1}$ and $C_{2}$ also determine the fine structure of the nature of any heterogeneous (shock) transition near to $z=0$. Clearly this can be non-monotonic. It is easy to identify this structure with the proximity of complex poles generated by the denominator of (89).

\section{Conclusions}

In this paper we have demonstrated three main new results: the use of hyperasymptotic expansions to seed a numerical solver with sufficiently accurate initial data; the occurrence and treatment of a higher order Stokes phenomena in a nonlinear ordinary differential equation; and the double resummation of transseries to produce doubly uniform solution for a higher order equation.

Although we have chosen to illustrate these results for a specific equation, the presence of these features may be common to higher order nonlinear differential equations with three or more exponential behaviours and requiring the presence of more than a single arbitrary constant.

The degree of ease of implementation of the above techniques will, of course, depend on the specific problem in question.

A rigorous proof of the results we have derived here seems desirable.

\section{Acknowledgements}

CJH is most grateful to the Research Institute for Mathematical Sciences, Kyoto University for their generous hospitality during which some of this work was undertaken. The authors also thank the referees for very helpful comments and suggestions for improving the presentation.

\section{Appendix A. Hyperasymptotics and optimal truncation}

For justification of the details in this section see [13], [14] and [15]. Here we will use the notation of section 2. Suppose that we are in a region bounded by the Stokes curves, a so-called Stokes region. Let $y_{0}(z ; \varepsilon)$ be the Borel-Laplace transform of $\hat{y}_{0}(z ; \varepsilon)$, that is, none of the exponentially small terms of the right-hand side of (10) are present in this Stokes region. Another way of defining this solution is via hyperasymptotic expansions. The level 0 hyperasymptotic expansion is just the optimal truncated expansion:

$$
y_{0}(z ; \varepsilon)=\sum_{s=0}^{N_{0}-1} a_{s}(z) \varepsilon^{s}+\mathcal{O}\left(e^{-N_{0}}\right),
$$

as $\varepsilon \rightarrow \infty$, where

$$
N_{0}=\min \left\{\left\lfloor\left|f_{j}(z) / \varepsilon\right|\right\rfloor: K_{j, 0} \neq 0\right\} .
$$


Note the requirement that $K_{j, 0} \neq 0$. Only the active small exponentials that can be switched on via a Stokes phenomenon affect the position and size of the least term. Conversely, note that these exponentials do not have to be actually present as a subdominant term in the expansion at a point $z$ to determine $N_{0}$, they only have to have the potential to be switched on as a Stokes line is crossed $\left(K_{j, 0} \neq 0\right)$.

From the properties of the Borel-Laplace transform ([13], [14] and [15]) the late terms of (A.1) will be linked to the early terms of the expansions involving the active small exponentials by a resurgence relation:

$$
a_{n}(z) \sim \sum_{j=1}^{2} \sum_{k=0}^{\infty} \frac{K_{j, k}}{2 \pi i} \sum_{s=0}^{\infty} \frac{b_{j, s}(z) \Gamma\left(n-s-k+\mu_{j}\right)}{\left(f_{j}(z)\right)^{n-s-k+\mu_{j}}}
$$

or more compactly with

$$
\beta_{j, s}(z)=\sum_{k=0}^{s} K_{j, k} b_{j, s-k}(z)
$$

we have

$$
a_{n}(z) \sim \sum_{j=1}^{2} \frac{1}{2 \pi i} \sum_{s=0}^{\infty} \frac{\beta_{j, s}(z) \Gamma\left(n-s+\mu_{j}\right)}{\left(f_{j}(z)\right)^{n-s+\mu_{j}}} .
$$

The level 1 hyperasymptotic expansion is then given

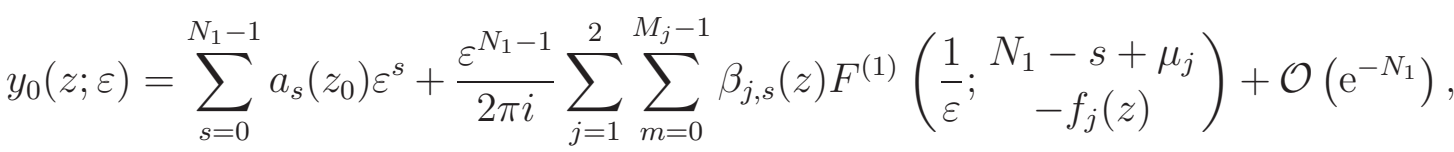

as $\varepsilon \rightarrow 0^{+}$, where

$$
M_{j}=\min \left\{\left\lfloor\left|f_{j}(z) / \varepsilon\right|\right\rfloor,\left\lfloor\left|\left(f_{j}(z)-f_{\ell}(z)\right) / \varepsilon\right|\right\rfloor: \ell \neq j\right\}
$$

and

$$
N_{1}=\min \left\{\left\lfloor f_{j}(z) / \varepsilon\right\rfloor+M_{j}: K_{j, 0} \neq 0\right\} .
$$

$F^{(1)}$ is a "universal hyperterminant", defined via

$$
F^{(1)}\left(\frac{1}{\varepsilon} ; \begin{array}{c}
M \\
\sigma
\end{array}\right)=\mathrm{e}^{M \pi i} \sigma^{1-M} \Gamma(M) U(1,2-M, \sigma / \varepsilon),
$$

where $U(a, c, z)$ is the confluent hypergeometric function ([23] §13.2). These hyperterminants deal with the Stokes phenomenon. Compare the re-expansion in (A.6) with the expansions in (17).

\section{References}

[1] S. Jonathan Chapman and David B. Mortimer. Exponential asymptotics and Stokes lines in a partial differential equation. Proc. R. Soc. Lond. Ser. A Math. Phys. Eng. Sci., 461(2060):23852421, 2005.

[2] C. J. Howls, P. J. Langman, and A. B. Olde Daalhuis. On the higher-order Stokes phenomenon. Proc. R. Soc. Lond. Ser. A Math. Phys. Eng. Sci., 460(2048):2285-2303, 2004. 
[3] M. V. Berry. Uniform asymptotic smoothing of Stokes's discontinuities. Proc. Roy. Soc. London Ser. A, 422(1862):7-21, 1989.

[4] H. L. Berk, William McCay Nevins, and K. V. Roberts. New Stokes' line in WKB theory. J. Math. Phys., 23(6):988-1002, 1982.

[5] Takashi Aoki, Takahiro Kawai, and Yoshitsugu Takei. On the exact steepest descent method: a new method for the description of Stokes curves. J. Math. Phys., 42(8):3691-3713, 2001.

[6] Takahiro Kawai, Tatsuya Koike, Yukihiro Nishikawa, and Yoshitsugu Takei. On the Stokes geometry of higher order Painlevé equations. Astérisque, (297):117-166, 2004. Analyse complexe, systèmes dynamiques, sommabilité des séries divergentes et théories galoisiennes. II.

[7] S. J. Chapman, C. J. Howls, J. R. King, and A. B. Olde Daalhuis. Why is a shock not a caustic? The higher-order Stokes phenomenon and smoothed shock formation. Nonlinearity, 20(10):2425-2452, 2007.

[8] M. V. Berry and C. J. Howls. Hyperasymptotics. Proc. Roy. Soc. London Ser. A, 430(1880):653668,1990 .

[9] M. V. Berry and C. J. Howls. Hyperasymptotics for integrals with saddles. Proc. Roy. Soc. London Ser. A, 434(1892):657-675, 1991.

[10] C. J. Howls. Hyperasymptotics for integrals with finite endpoints. Proc. Roy. Soc. London Ser. A, 439(1906):373-396, 1992.

[11] C. J. Howls. Hyperasymptotics for multidimensional integrals, exact remainder terms and the global connection problem. Proc. Roy. Soc. London Ser. A, 453(1966):2271-2294, 1997.

[12] C. J. Howls and A. B. Olde Daalhuis. Hyperasymptotic solutions of inhomogeneous linear differential equations with a singularity of rank one. R. Soc. Lond. Proc. Ser. A Math. Phys. Eng. Sci., 459(2038):2599-2612, 2003.

[13] A. B. Olde Daalhuis. Hyperasymptotic solutions of higher order linear differential equations with a singularity of rank one. R. Soc. Lond. Proc. Ser. A Math. Phys. Eng. Sci., 454(1968):1-29, 1998.

[14] A. B. Olde Daalhuis. Hyperasymptotics for nonlinear ODEs. I. A Riccati equation. Proc. R. Soc. Lond. Ser. A Math. Phys. Eng. Sci., 461(2060):2503-2520, 2005.

[15] A. B. Olde Daalhuis. Hyperasymptotics for nonlinear ODEs. II. The first Painlevé equation and a second-order Riccati equation. Proc. R. Soc. Lond. Ser. A Math. Phys. Eng. Sci., 461(2062):3005-3021, 2005.

[16] A. B. Olde Daalhuis. On higher-order Stokes phenomena of an inhomogeneous linear ordinary differential equation. J. Comput. Appl. Math., 169(1):235-246, 2004.

[17] O. Costin and R. D. Costin. On the formation of singularities of solutions of nonlinear differential systems in antistokes directions. Invent. Math., 145(3):425-485, 2001.

[18] K. L. Adams, J. R. King, and R. H. Tew. Beyond-all-orders effects in multiple-scales asymptotics: travelling-wave solutions to the Kuramoto-Sivashinsky equation. J. Engrg. Math., 45(3-4):197226, 2003. Practical asymptotics, II.

[19] S. J. Chapman, J. R. King, and K. L. Adams. Exponential asymptotics and Stokes lines in nonlinear ordinary differential equations. R. Soc. Lond. Proc. Ser. A Math. Phys. Eng. Sci., 454(1978):2733-2755, 1998.

[20] Ovidiu Costin. On Borel summation and Stokes phenomena for rank-1 nonlinear systems of ordinary differential equations. Duke Math. J., 93(2):289-344, 1998.

[21] A. B. Olde Daalhuis. Hypergeometric function. In NIST handbook of mathematical functions, pages 383-401. U.S. Dept. Commerce, Washington, DC, 2010.

[22] Christopher J. Howls. On the use of Z-transforms in the summation of transseries for partial differential equations. In Algebraic analysis of differential equations from microlocal analysis to exponential asymptotics, pages 133-141. Springer, Tokyo, 2008.

[23] Digital Library of Mathematical Functions. National institute of standards and technology. from http://dlmf.nist.gov/, 2010. 